\title{
The influence of wall permeability on turbulent channel flow
}

\author{
By W. P. BREUGEM ${ }^{1,3}$, B. J. BOERSMA ${ }^{1}$ \\ AND R. E. UITTENBOGAARD ${ }^{2,1}$ \\ ${ }^{1}$ J. M. Burgers Center for Fluid Dynamics, Delft University of Technology, \\ Leeghwaterstraat 21, 2628 CA Delft, The Netherlands \\ ${ }^{2}$ WL | Delft Hydraulics, PO Box 177, 2600 MH Delft, The Netherlands \\ ${ }^{3}$ Royal Netherlands Meteorological Institute (KNMI), PO Box 201, \\ 3730 AE De Bilt, The Netherlands
}

(Received 25 November 2004 and in revised form 25 January 2006)

Direct numerical simulations (DNS) have been performed of turbulent flow in a plane channel with a solid top wall and a permeable bottom wall. The permeable wall is a packed bed, which is characterized by the mean particle diameter and the porosity. The main objective is to study the influence of wall permeability on the structure and dynamics of turbulence. The flow inside the permeable wall is described by means of volume-averaged Navier-Stokes equations. Results from four simulations are shown, for which only the wall porosity $\left(\epsilon_{c}\right)$ is changed. The Reynolds number based on the thickness of the boundary layer over the permeable wall and the friction velocity varies from $R e_{\tau}^{p}=176$ for $\epsilon_{c}=0$ to $R e_{\tau}^{p}=498$ for $\epsilon_{c}=0.95$. The influence of wall permeability can be characterized by the permeability Reynolds number, $R e_{K}$, which represents the ratio of the effective pore diameter to the typical thickness of the viscous sublayers over the individual wall elements. For small $R e_{K}$, the wall behaves like a solid wall. For large $R e_{K}$, the wall is classified as a highly permeable wall near which viscous effects are of minor importance. It is observed that streaks and the associated quasi-streamwise vortices are absent near a highly permeable wall. This is attributed to turbulent transport across the wall interface and the reduction in mean shear due to a weakening of, respectively, the wall-blocking and the wall-induced viscous effect. The absence of streaks is consistent with a decrease in the peak value of the streamwise root mean square (r.m.s.) velocity normalized by the friction velocity at the permeable wall. Despite the increase in the peak values of the spanwise and wall-normal r.m.s. velocities, the peak value of the turbulent kinetic energy is therefore smaller. Turbulence near a highly permeable wall is dominated by relatively large vortical structures, which originate from a Kelvin-Helmholtz type of instability. These structures are responsible for an exchange of momentum between the channel and the permeable wall. This process contributes strongly to the Reynolds-shear stress and thus to a large increase in the skin friction.

\section{Introduction}

Turbulent flows over permeable walls, i.e. rigid porous walls with interconnected pores through which fluid may flow, are encountered in a wide range of problems. Examples are: flows in oil wells, catalytic reactors, heat exchangers of open-cell metal foam (Lu, Stone \& Ashby 1998), and porous river beds (Vollmer et al. 2002). To some extent, densely built-up urban areas and plant canopies can be considered as 
permeable wall layers as well. Related research topics are dispersion of pollutants in metropoles, the exchange of energy and oxide and carbon dioxide between forests and the atmosphere (Finnigan 2000), and the propagation of forest fires (Séro-Guillaume \& Margerit 2002).

Despite its relevance to the aforementioned applications, only a few experimental studies report on the effect of wall permeability on turbulence. Zagni \& Smith (1976) conducted experiments on open-channel flow over permeable beds composed of spheres. It was found that the friction factor was higher than for flows over impermeable walls with the same surface roughness. Furthermore, after having reached a constant value, at typically $R e=O\left(10^{5}\right)$ the friction factor showed a tendency to increase again with Reynolds number. Also Kong \& Schetz (1982) reached the conclusion that in their experiments wall permeability alone could be responsible for an increase in skin friction by as much as $30-40 \%$ relative to an impermeable wall with similar surface roughness. A rise in friction factor at high Reynolds numbers was also observed by Zippe \& Graf (1983) in wind-tunnel experiments on boundary-layer flow over a permeable bed composed of grains. Zagni \& Smith (1976) attributed the increase in friction factor to additional energy dissipation caused by exchange of momentum across the bed interface. Evidence of this exchange has been provided by experiments of Ruff \& Gelhar (1972) on turbulent flow in a pipe lined with highly porous foam.

The experiments mentioned above clearly indicate that the effect of wall permeability on turbulence is different from wall roughness. This implies that wall permeability alters the structure and dynamics of turbulence. In this paper we aim to improve our understanding of this alteration by means of direct numerical simulation (DNS) of turbulent flow over a permeable wall. Unlike experiments, in DNS it is relatively easy to isolate the effect of wall permeability from wall roughness. Furthermore, these simulations provide very detailed information on the flow field both above and inside a permeable wall, which is difficult to obtain from measurements. In literature two different methods can be found for simulating flow over and through a permeable wall.

The first and computationally most simple method is the specification of boundary conditions that incorporate the effect of wall permeability. This approach was followed by Hahn, Je \& Choi (2002) in DNS of turbulent flow in a plane channel with permeable walls. The boundary conditions used were similar to those proposed by Beavers \& Joseph (1967) for laminar flow over a permeable wall, and allow for a slipvelocity at the wall. The wall-normal velocity was put to zero, which is, however, not realistic for high wall permeabilities at which exchange of momentum across the wall interface takes place (Ruff \& Gelhar 1972). As pointed out by Hahn, Je \& Choi (2002), a wall can be classified as highly permeable when the permeability Reynolds number $R e_{K} \equiv \sqrt{K} u_{\tau} / v \gg 1$, where $K$ is the wall permeability of the order of the square of the characteristic pore diameter, $v$ is the kinematic viscosity and $u_{\tau}$ is the wall friction velocity. Therefore, for an accurate description of flow over a permeable wall at high $R e_{K}$, the flow inside the wall itself also has to be described.

The second method is the continuum approach where the flow inside the permeable wall is modelled as a continuum, which is coupled to the flow over the wall. The theoretical basis for this approach is provided by the volume-averaging method (Whitaker 1999). In this method the flow is averaged over a small spatial volume with dimensions sufficiently large to smooth inhomogeneities at pore scales, but on the other hand sufficiently small to retain the flow dynamics of interest. The volumeaveraged flow field is governed by the volume-averaged Navier-Stokes (VANS) equations (Whitaker 1996). In order to solve the VANS equations, closures are required for 
the subfilter-scale stress and the drag force. The continuum approach has been used in a number of recent large-eddy simulation (LES) studies of flows over forests (e.g. Shaw \& Schumann 1992; Dwyer, Patton \& Shaw 1997; Watanabe 2004). Other studies, in which a Reynolds-averaged form of the VANS equations was used, considered not only flow over vegetation (Wilson 1988; Uittenbogaard 2003), but also flow over a permeable wall layer with a porosity significantly lower than that of vegetation (De Lemos \& Pedras 2000; Silva \& De Lemos 2003).

In a recent publication (Breugem \& Boersma 2005), we verified that the VANS equations can be used for an accurate simulation of turbulent flow over and through a permeable wall. The VANS equations were solved in a DNS of turbulent channel flow with a lower permeable wall consisting of a three-dimensional Cartesian grid of cubes. The turbulence statistics agreed very well with the results of a different DNS in which, by means of the standard Navier-Stokes equations, the flow field in between the cubes was fully resolved. This gives us confidence for using the VANS equations in the present study where we consider again flow in a plane channel with a lower permeable wall. Different from the grid of cubes in our previous study, the permeable wall in the present study is a packed bed, which is encountered in many applications. In the DNS, the packed bed is characterized by the porosity and the mean particle diameter. In order to isolate the effect of wall permeability from wall roughness, we consider packed beds with relatively high wall porosities and small mean particle diameters. Results from four simulations will be shown, each with a different porosity.

This paper is organized as follows. Section 2 discusses the continuum approach for flows through porous media. The next section deals with the coupling between the flow in the channel and the flow inside the permeable wall. In $\S 4$, a discussion is given of the implications of wall permeability for the scaling of turbulence. Section 5 deals with the numerical method. The DNS results are presented in $\S 6$. In $\S 7$, the results are summarized and discussed.

\section{Continuum approach for flows in porous media}

In this section, we briefly discuss the continuum approach for flows in porous media. See Breugem (2004) for a detailed discussion.

The first step in the derivation of the governing equations for the volume-averaged flow is the definition of the superficial volume average, denoted by $\langle\cdots\rangle^{s}$ :

$$
\langle\boldsymbol{u}\rangle_{\boldsymbol{x}}^{s} \equiv \int_{V} \gamma(\boldsymbol{r}) m(\boldsymbol{y}) \boldsymbol{u}(\boldsymbol{r}) \mathrm{d} V
$$

where the subscript $\boldsymbol{x}$ means that the volume average is evaluated at the centroid $\boldsymbol{x}$ of the averaging volume $V, \boldsymbol{y}=\boldsymbol{r}-\boldsymbol{x}$ is the relative position vector, $\gamma$ is the phaseindicator function that equals unity when $\boldsymbol{r}$ points in the fluid phase and zero when $\boldsymbol{r}$ points in the solid phase, and $m$ is a weighting function. The volume-averaging technique is illustrated in figure 1 . Notice that the volume-averaging operator acts as a filter, which passes information only on the large-scale structure of the flow field. Furthermore, we note that the volume-averaged flow field is continuous in the sense that it is defined both in the fluid and the solid phase, provided of course that the averaging volume is sufficiently large. This is the basis of the continuum approach for flows in porous media.

In principle, the weighting function can be chosen freely, but it is desirable that the volume-averaged flow field contains negligible variations on scales smaller than the 


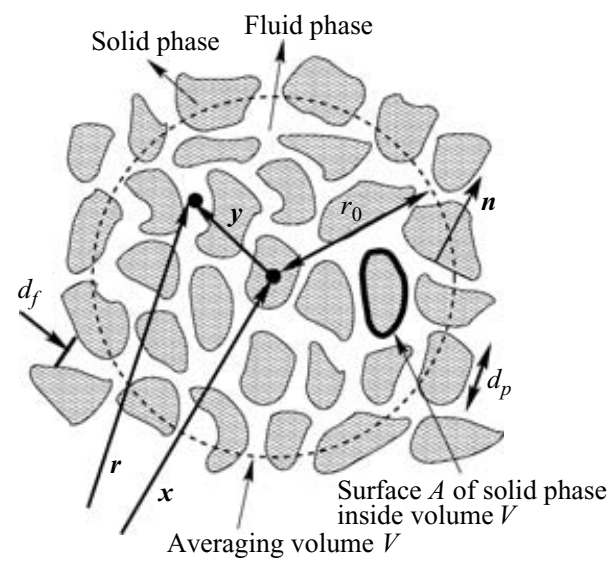

FIGURE 1. Illustration of the volume-averaging technique for a disordered porous medium.

dimensions of the averaging volume. A customary averaging volume for a disordered porous medium as sketched in figure 1, is a sphere with radius $r_{0}$ and a top-hat distribution for the weighting function (Quintard \& Whitaker 1994):

$$
m(\boldsymbol{y})= \begin{cases}3 /\left(4 \pi r_{0}^{3}\right), & |\boldsymbol{y}| \leqslant r_{0}, \\ 0, & |\boldsymbol{y}|>r_{0} .\end{cases}
$$

The velocity at a certain point in the porous medium can be decomposed into a contribution from the volume-averaged velocity at this point and a subfilter-scale velocity $\tilde{\boldsymbol{u}}$ according to (Gray 1975):

$$
\boldsymbol{u}=\langle\boldsymbol{u}\rangle+\tilde{\boldsymbol{u}}
$$

where $\langle\boldsymbol{u}\rangle \equiv\langle\boldsymbol{u}\rangle^{s} / \epsilon$ is the intrinsic volume average and $\epsilon$ is the porosity. The latter is defined according to:

$$
\epsilon(\boldsymbol{x}) \equiv \int_{V} \gamma(\boldsymbol{r}) m(\boldsymbol{y}) \mathrm{d} V .
$$

Later on in this paper we will also make use of a temporal decomposition according to (Tennekes \& Lumley 1999):

$$
\boldsymbol{u}=\overline{\boldsymbol{u}}+\boldsymbol{u}^{\prime}
$$

where the overbar denotes the Reynolds- or ensemble-averaged value and the prime denotes the deviation from the Reynolds-averaged value. It is easy to show that the ensemble- and spatial-averaging operators commute (Pedras \& De Lemos 2001): $\overline{\langle\boldsymbol{u}\rangle}=\langle\overline{\boldsymbol{u}}\rangle,\langle\boldsymbol{u}\rangle^{\prime}=\left\langle\boldsymbol{u}^{\prime}\right\rangle, \overline{\tilde{\boldsymbol{u}}}=\tilde{\overline{\boldsymbol{u}}}$ and $\tilde{\boldsymbol{u}}^{\prime}=\widetilde{\boldsymbol{u}^{\prime}}$.

The spatial averaging theorem (Whitaker 1969) relates the volume average of a spatial derivative to the spatial derivative of the volume average:

$$
\langle\nabla p\rangle_{\boldsymbol{x}}^{s}=\nabla\langle p\rangle_{\boldsymbol{x}}^{s}+\int_{A} m(\boldsymbol{y}) \boldsymbol{n} p(\boldsymbol{r}) \mathrm{d} A,
$$

where $A$ is the contact area between the fluid and the solid phase inside the averaging volume $V$, and $\boldsymbol{n}$ is the unit normal at $A$ that points from the fluid into the solid phase, see figure 1. 
Application of the spatial filter (2.1) and the spatial averaging theorem (2.6) to the Navier-Stokes equations yields the volume-averaged Navier-Stokes (VANS) equations (Whitaker 1996):

$$
\begin{aligned}
\frac{\partial\langle\boldsymbol{u}\rangle}{\partial t}+\frac{1}{\epsilon} \nabla \cdot[\epsilon\langle\boldsymbol{u}\rangle\langle\boldsymbol{u}\rangle]+\frac{1}{\epsilon} \nabla \cdot[\epsilon \boldsymbol{\tau}] & =-\frac{1}{\epsilon \rho} \nabla[\epsilon\langle p\rangle]+\frac{\nu}{\epsilon} \nabla^{2}[\epsilon\langle\boldsymbol{u}\rangle]+\boldsymbol{f}, \\
\nabla \cdot[\epsilon\langle\boldsymbol{u}\rangle] & =0,
\end{aligned}
$$

where $\boldsymbol{\tau}$ is the subfilter-scale stress, which in the LES literature is known as the subgrid-scale stress, and $f$ is the drag force per unit mass that the solid phase exerts on the fluid phase. The expressions for $\tau$ and $f$ are given by:

$$
\begin{aligned}
\boldsymbol{\tau} & \equiv\langle\boldsymbol{u} \boldsymbol{u}\rangle-\langle\boldsymbol{u}\rangle\langle\boldsymbol{u}\rangle \approx\langle\tilde{\boldsymbol{u}} \tilde{\boldsymbol{u}}\rangle \\
\boldsymbol{f} & \equiv \frac{1}{\epsilon} \int_{A} m \boldsymbol{n}\left[-\frac{p}{\rho} \boldsymbol{I}+v \nabla \boldsymbol{u}\right] \mathrm{d} A \\
& \approx \frac{1}{\epsilon} \int_{A} m \boldsymbol{n}\left[-\frac{\tilde{p}}{\rho} \boldsymbol{I}+v \nabla \tilde{\boldsymbol{u}}\right] \mathrm{d} A+\frac{\langle p\rangle}{\rho \epsilon} \nabla \epsilon-\frac{v}{\epsilon} \nabla \epsilon \cdot \nabla\langle\boldsymbol{u}\rangle,
\end{aligned}
$$

where the approximations in the last two equations are valid when $\langle\langle\boldsymbol{u}\rangle\rangle \approx\langle\boldsymbol{u}\rangle$.

In order to solve the VANS equations, closures are required for the subfilter-scale stress and the drag force in terms of the volume-averaged flow quantities. In Appendix A, we argue that, in porous media, subfilter-scale dispersion is normally negligible with respect to the drag force and/or the Reynolds-shear stress of the volume-averaged flow field.

Whitaker (1996) gives theoretical support to the following customary parameterization of the drag force:

$$
\frac{1}{\epsilon} \int_{A} m \boldsymbol{n}\left[-\frac{\tilde{p}}{\rho} \boldsymbol{I}+v \nabla \tilde{\boldsymbol{u}}\right] \mathrm{d} A=-v \boldsymbol{K}^{-1} \epsilon\langle\boldsymbol{u}\rangle-v \boldsymbol{K}^{-1} \boldsymbol{F} \epsilon\langle\boldsymbol{u}\rangle,
$$

where $\boldsymbol{K}$ and $\boldsymbol{F}$ are, respectively, the permeability and the Forchheimer tensor. The first term on the right-hand side of (2.8) represents the drag force in the limit of Stokes flow in the pores, whereas the second term is a correction for inertial effects at higher Reynolds numbers. In general, the permeability tensor depends only on the geometry of the porous medium. The Forchheimer tensor depends on the Reynolds number $|\langle\boldsymbol{u}\rangle| d_{f} / \nu$, with $d_{f}$ the typical pore diameter, on the geometrical parameters of the porous medium and on the orientation of the solid obstacles relative to the direction of the volume-averaged flow. Generally valid expressions for the permeability and the Forchheimer tensor do not exist, as they are strongly related to the geometry of the porous medium and the Reynolds number. They must be determined from experiments or numerical calculations of flow through a representative region of the porous medium. Numerical calculations of the permeability and the Forchheimer tensor for several geometries are presented by Zick \& Homsy (1982), Larson \& Higdon (1986, 1987), Sahraoui \& Kaviany (1992), Ma \& Ruth (1993), Lee \& Yang (1997) and Breugem, Boersma \& Uittenbogaard (2004). A few references to experiments are MacDonald et al. (1979), Fand et al. (1987), Kececioglu \& Jiang (1994) and Lage, Antohe \& Nield (1997). For flows through packed beds, which are considered in the present study, a widely used relation for the drag force is the modified Ergun equation (Bird, Stewart \& Lightfoot 2002). This equation can be written in the form of (2.8) 


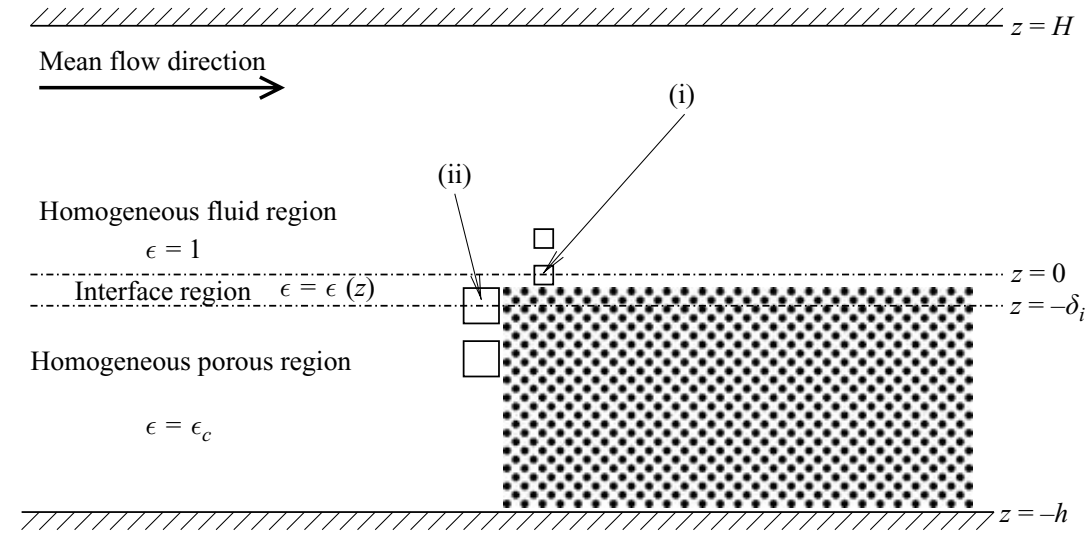

FIGURE 2. Sketch of the flow geometry. (i) Centroid averaging volume that marks the beginning of the interface region; (ii) centroid averaging volume that marks the end of the interface region.

with the following relations for the permeability and the Forchheimer tensor:

$$
\begin{aligned}
\boldsymbol{K} & =\frac{d_{p}{ }^{2} \epsilon^{3}}{180(1-\epsilon)^{2}} \boldsymbol{I}, \\
\boldsymbol{F} & =\tilde{F}|\langle\boldsymbol{u}\rangle| \boldsymbol{I}, \quad \tilde{F} \equiv \frac{\epsilon}{100(1-\epsilon)} \frac{d_{p}}{v},
\end{aligned}
$$

where $d_{p} \equiv 6 V_{p} / A_{p}$ is the mean particle diameter with $V_{p}$ the volume and $A_{p}$ the surface area of the solid obstacles. The coefficients in (2.9a) and (2.9b) were determined from many experiments with different materials, in which the particle sizes, the porosity and the Reynolds number were varied (MacDonald et al. 1979).

\section{Flow geometry and drag model for the interface region}

In our study, we consider the flow geometry as sketched in figure 2 . The upper wall is a solid wall. The lower wall is a homogeneous packed bed. The lower side of the packed bed is bounded by a solid wall. Following Breugem \& Boersma (2005), we distinguish between three regions.

(a) The homogeneous fluid region or channel region between $z=0$ and $z=H$ in which the porosity is equal to unity.

(b) A small interface region between $z=-\delta_{i}$ and $z=0$, characterized by a spatially varying porosity.

(c) The homogeneous porous region between $z=-h$ and $z=-\delta_{i}$, with a constant porosity $\left(\epsilon=\epsilon_{c}\right)$.

Below, we discuss how the flow is described in each region. As already mentioned in $\S 1$, the approach followed here has been successfully validated in Breugem \& Boersma (2005).

\subsection{Homogeneous porous region}

Based on the arguments given in Appendix A, in the homogeneous porous region subfilter-scale dispersion can be neglected. The drag force is modelled by means of the Ergun equation for packed beds. Combination of $(2.7 a),(2.7 d)$ and (2.8), yields 
the following transport equation for the flow in the homogeneous porous region:

$$
\frac{\partial\langle\boldsymbol{u}\rangle}{\partial t}+\nabla \cdot\langle\boldsymbol{u}\rangle\langle\boldsymbol{u}\rangle=-\frac{1}{\rho} \nabla\langle p\rangle+v \nabla^{2}\langle\boldsymbol{u}\rangle-v \boldsymbol{K}^{-1}(\boldsymbol{I}+\boldsymbol{F}) \epsilon\langle\boldsymbol{u}\rangle,
$$

where $\boldsymbol{K}$ and $\boldsymbol{F}$ are given by (2.9a) and (2.9b), respectively.

\subsection{Channel region}

In the homogeneous fluid region, there are no solid obstacles $(\epsilon=1)$ and hence the drag force exerted on the flow is zero. Thus the VANS equations reduce to the LES equations. The subfilter-scale dispersion term is negligible only when the filter length is sufficiently small. This may not be the case when the same filter length is used for the channel region as for the homogeneous porous region. In our DNS, we aim to completely resolve the flow field in the channel region. This can be accomplished by assuming that in the channel region the filter length is set by the computational mesh spacing $\Delta$, such that, provided that $\Delta$ is sufficiently small, subfilter-scale dispersion is negligible. Consequently, in the channel region, $\langle\boldsymbol{u}\rangle=\boldsymbol{u}$ holds and the VANS equations reduce to the standard Navier-Stokes equations, i.e. (3.1) without the drag term.

\subsection{Interface region}

Like for the homogeneous porous and the homogeneous fluid region, it is assumed that subfilter-scale dispersion can be neglected in the interface region too. The drag force cannot be neglected, however. It is modelled by means of the Ergun equation in combination with a variable-porosity model, which ensures that the drag force is continuous over the interface region. A similar approach has been used by OchoaTapia \& Whitaker (1995) to study the laminar flow in a plane channel with one permeable wall and one solid wall and by Vafai (1984) to analyse the channelling effect in a porous medium near a solid boundary. Below we discuss the variableporosity model in more detail.

By definition the upper boundary of the interface region $(z=0$ in figure 2$)$ is the position of the centroid of the averaging volume for which the lower part of the averaging volume just touches the solid obstacles in the top region of the permeable wall. The porosity is still equal to unity. When the averaging volume is shifted into the permeable wall, the porosity gradually decreases, until it reaches the value of $\epsilon=\epsilon_{c}$ for $z=-\delta_{i}$. In principle, when the topology of the permeable wall is known, the porosity in the interface region can be calculated from (2.4), but this is not a straightforward calculation. The porosity is only a function of the height $z$, equal to unity at $z=0$ and equal to $\epsilon_{c}$ at $z=-\delta_{i}$. Furthermore, it is required that the VANS equations are continuous at the boundaries of the interface region with the homogeneous fluid and the homogeneous porous region. This leads to four additional constraints for the porosity, because it implies that the porosity must have continuous first and second-order derivatives. The simplest profile for the porosity, which satisfies all six constraints, is a fifth-order polynomial in $z$ :

$-\delta_{i} \leqslant z \leqslant 0: \quad \epsilon(z)=-6\left(\epsilon_{c}-1\right)\left(\frac{z}{\delta_{i}}\right)^{5}-15\left(\epsilon_{c}-1\right)\left(\frac{z}{\delta_{i}}\right)^{4}-10\left(\epsilon_{c}-1\right)\left(\frac{z}{\delta_{i}}\right)^{3}+1$.

The above model for the porosity requires a specification of the thickness $\delta_{i}$ of the interface region. As illustrated in figure $2, \delta_{i}$ is of the order of the filter length. 
Combining $(2.7 a),(2.7 d)$ and (2.8) yields the following transport equation for the flow in the interface region:

$$
\begin{aligned}
\frac{\partial\langle\boldsymbol{u}\rangle}{\partial t}+\frac{1}{\epsilon} \nabla \cdot[\epsilon\langle\boldsymbol{u}\rangle\langle\boldsymbol{u}\rangle]= & -\frac{1}{\rho} \nabla\langle p\rangle+v \nabla^{2}\langle\boldsymbol{u}\rangle+\frac{v}{\epsilon} \nabla \epsilon \cdot \nabla\langle\boldsymbol{u}\rangle \\
& +\frac{v}{\epsilon}\left[\nabla^{2} \epsilon\right]\langle\boldsymbol{u}\rangle-v \boldsymbol{K}^{-1}[\boldsymbol{I}+\boldsymbol{F}] \epsilon\langle\boldsymbol{u}\rangle,
\end{aligned}
$$

with the porosity described by (3.2), and $\boldsymbol{K}$ and $\boldsymbol{F}$ given by (2.9a) and (2.9b), respectively.

We remark that the governing equations given in this section do not contain any explicit information on the spatial filter by which they were derived from the Navier-Stokes equations. In a way, we could say that some information on the filter is 'hidden' in the closure for the drag force, the neglect of the subfilter-scale stress, and the prescribed porosity profile and thickness of the interface region. However, we emphasize that no explicit information on the filter is required to solve the governing equations.

To simplify the notation, from now on we will omit the brackets denoting the intrinsic volume average of a quantity.

\section{Implications of wall permeability for scaling of turbulence}

In this section, the implications of wall permeability on the scaling of turbulence are discussed. In the DNS, the permeable wall is characterized by three different length scales: the thickness $h$, the mean particle diameter $d_{p}$ and the square root of the permeability $\sqrt{K_{c}} \equiv \sqrt{K\left(\epsilon=\epsilon_{c}\right)}$. The latter scale can be interpreted as the effective pore diameter. The thickness $\delta_{i}$ of the interface region is related to the filter length and hence to $d_{p}$, and is thus not an independent length scale. To avoid any influence of the impermeable wall at $z=-h$ below the porous medium on the flow in the channel, it is required that $h$ is much larger than the penetration depth of turbulence inside the permeable wall. In that case, $h$ is no longer a relevant length scale for the flow. This is in contrast to flow over vegetation where the penetration depth of turbulence is usually of the same order as the whole vegetation height (Finnigan 2000).

Permeable walls are usually also rough. In principle, the additional effect of surface roughness could be quantified by comparing the flow over the permeable and rough wall with the flow over an impermeable wall with the same surface roughness. In literature, it is common practice to characterize a rough wall by the typical height of the roughness elements, which in our case is the mean particle diameter $d_{p}$. The effect of roughness depends on the roughness Reynolds number, defined according to:

$$
R e_{d} \equiv \frac{d_{p} u_{\tau}}{v}
$$

Based on Nikuradse's experiments on flows through circular pipes with a sandroughened wall, Hinze (1975, p. 635) defines a fully rough wall, for which the thickness of the viscous sublayer is much less than the height of the roughness elements, by the condition that $R e_{d}>55$. According to Hinze's classification, the effect of roughness is negligible when $R e_{d}<5$. Although this classification is based on experiments with rough and simultaneously impermeable walls, it gives a clue to the importance of roughness for cases where the wall is not only rough, but also permeable. 
Similarly to (4.1), the effect of wall permeability depends on the permeability Reynolds number, defined according to:

$$
R e_{K} \equiv \frac{\sqrt{K_{c}} u_{\tau}}{v} .
$$

This number can be interpreted as the ratio of the effective pore diameter to the typical length scale of the viscous sublayers over the individual wall elements. Wall permeability is expected to have only an influence on the turbulent flow when this ratio is sufficiently large. For small $R e_{K}$, the discontinuous viscous sublayers over the wall elements are relatively thick and they coalesce and form a continuous classical viscous sublayer covering the complete wall. This sublayer in turn prevents penetration of turbulent eddies into the wall. Thus, for small $R e_{K}$ a permeable wall is effectively impermeable.

In this work, we aim to study exclusively the effect of wall permeability. Therefore it is required that $R e_{d}$ is sufficiently small and that $R e_{K}$ is sufficiently large. This can be accomplished by choosing a small value for $d_{p}$ and a high value for $\epsilon_{c}$.

\subsection{Channel region}

In the channel region of our flow geometry, two boundary layers can be distinguished: one above the permeable wall and one below the solid top wall. The border between the two boundary layers corresponds to the location of the mean velocity maximum or, equivalent to this, the height $z=\delta_{w}$ at which the total shear stress $\tau_{x z}$ is equal to zero. An expression for the total shear stress is found from integration of the Reynolds-averaged momentum equation:

$$
\frac{\tau_{x z}}{\rho} \equiv-\overline{u^{\prime} w^{\prime}}+\nu \frac{\partial \bar{u}}{\partial z}=-\left[\left(u_{\tau}^{p}\right)^{2}+\left(u_{\tau}^{t}\right)^{2}\right] \frac{z}{H}+\left(u_{\tau}^{p}\right)^{2}, \quad 0 \leqslant z \leqslant H,
$$

where $u_{\tau}^{p} \equiv \sqrt{\tau_{x z}(0) / \rho}$ is the friction velocity at the permeable wall and $u_{\tau}^{t} \equiv$ $\sqrt{-\tau_{x z}(H) / \rho}$ is the friction velocity at the top wall. An expression for $\delta_{w}$ can then be obtained by substituting $\tau_{x z}\left(\delta_{w}\right) \equiv 0$ into the above equation:

$$
\frac{\delta_{w}}{H}=\frac{\left(u_{\tau}^{p}\right)^{2}}{\left(u_{\tau}^{p}\right)^{2}+\left(u_{\tau}^{t}\right)^{2}} .
$$

According to the well-known classic theory (Hinze 1975; Tennekes \& Lumley 1999) for turbulent flow over a solid wall, in the boundary layer at the top wall, an inner and an outer region can be distinguished. The characteristic velocity scale for both regions is the friction velocity $u_{\tau}^{t}$ at the top wall. Characteristic length scales are $v / u_{\tau}^{t}$ and $H-\delta_{w}$ for the inner and the outer regions, respectively.

As for the boundary layer at the top wall, we expect also an inner and an outer region in the boundary layer above the permeable wall. The characteristic velocity scale for both regions is the friction velocity $u_{\tau}^{p}$ at the permeable wall. The boundarylayer thickness $\delta_{w}$ is a typical length scale for the outer region. For $R e_{K} \gg 1$ it can be expected that the effect of viscosity on the flow is negligible. As we have assumed that $R e_{d}$ is small, the single relevant turbulence length scale left over for the inner region is $\sqrt{K_{c}}$.

Just as for turbulent flows above solid and rough walls, for a sufficiently high Reynolds number $u_{\tau}^{p} \delta_{w} / \nu$, a logarithmic layer can be expected in the boundary layer 
above the permeable wall as well. A customary parameterization of the log law reads:

$$
\frac{\bar{u}}{u_{\tau}^{p}}=\frac{1}{\lambda} \ln \left(\frac{z+d}{z_{0}}\right),
$$

where $d$ is the displacement height, $z_{0}$ the equivalent roughness height, and $\lambda$ is equivalent to the von Kármán constant of approximately 0.4 .

\subsection{Homogeneous porous region}

We now take a closer look at the scaling of turbulent flow inside a permeable wall. In the homogeneous porous region the Reynolds-averaged VANS equations reduce to:

$$
\begin{aligned}
& 0=-\frac{1}{\rho} \frac{\partial \bar{p}}{\partial x}-\frac{\partial \overline{u^{\prime} w^{\prime}}}{\partial z}+v \frac{\partial^{2} \bar{u}}{\partial z^{2}}-\frac{v \epsilon_{c}}{K_{c}} \bar{u}-\frac{v \epsilon_{c} \tilde{F}_{c}}{K_{c}} \overline{|\boldsymbol{u}| u}, \\
& 0=-\frac{1}{\rho} \frac{\partial \bar{p}}{\partial z}-\frac{\partial \overline{w^{\prime} w^{\prime}}}{\partial z}-\frac{\nu \epsilon_{c} \tilde{F}_{c}}{K_{c}} \overline{|\boldsymbol{u}| w},
\end{aligned}
$$

where the subscript $c$ refers to the constant porosity in this region, and $\tilde{F}_{c} \equiv \tilde{F}\left(\epsilon=\epsilon_{c}\right)$ with $\tilde{F}$ defined in $(2.9 b)$. For small $R e_{K},(4.6 a)$ and $(4.6 b)$ reduce to the Brinkman equations (Brinkman 1948):

$$
\begin{aligned}
& 0=-\frac{1}{\rho} \frac{\partial \bar{p}}{\partial x}+v \frac{\partial^{2} \bar{u}}{\partial z^{2}}-\frac{v \epsilon_{c}}{K_{c}} \bar{u}, \\
& 0=-\frac{1}{\rho} \frac{\partial \bar{p}}{\partial z} .
\end{aligned}
$$

The solution is a decaying exponential function, which matches the velocity $U_{i}$ at $z=-\delta_{i}$ to the Darcy velocity $U_{d}$ further down inside the permeable wall:

$$
\begin{aligned}
\bar{u} & =U_{d}+\left(U_{i}-U_{d}\right) \exp \left[z \sqrt{\frac{\epsilon_{c}}{K_{c}}}\right], \quad z \leqslant-\delta_{i}, \\
U_{d} & =-\frac{1}{\mu} \frac{K_{c}}{\epsilon_{c}} \frac{\partial \bar{p}}{\partial x} .
\end{aligned}
$$

Equation (4.7a) expresses the balance between viscous diffusion of momentum from the channel into the permeable wall and the Darcy drag force. For a high value of $R e_{K}$, turbulent diffusion and Forchheimer drag become important. However, in that case it is difficult to obtain an analytical expression for the velocity profile from $(4.6 a)$. For flow in plant canopies the mean velocity is usually parameterized by an exponential function, and, as will be shown in $\S 6.1$, this appears to be a good approximation:

$$
\bar{u} \approx U_{d}+\left(U_{i}-U_{d}\right) \exp \left[\alpha z \sqrt{\frac{\epsilon_{c}}{K_{c}}}\right], \quad z \leqslant-\delta_{i},
$$

where $\alpha$ is an empirical coefficient. The velocity $U_{d}$ is found from the balance between the pressure gradient and the two drag terms in $(4.6 a)$ :

$$
U_{d}=\frac{\left(1-\frac{4}{\mu} \frac{K_{c}}{\epsilon_{c}} \frac{\partial \bar{p}}{\partial x} \tilde{F}_{c}\right)^{1 / 2}-1}{2 \tilde{F}_{c}} .
$$

If $R e_{K}$ is small, then (4.9a) reduces to (4.8a) with the value of $\alpha$ equal to unity. For very large $R e_{K}$, turbulent diffusion is balanced solely by Forchheimer drag. In this 
case the value for $\alpha$ can be estimated from:

$$
\frac{\left[-\frac{\partial \overline{u^{\prime} w^{\prime}}}{\partial z}\right]}{\left[-\frac{\nu \epsilon_{c} \tilde{F}_{c}}{K_{c}} \overline{|\boldsymbol{u}| u}\right]} \sim \frac{\left[\alpha \sqrt{\frac{\epsilon_{c}}{K_{c}}}\left(u_{\tau}^{p}\right)^{2}\right]}{\left[v \frac{\epsilon_{c}}{K_{c}} \tilde{F}_{c}\left(u_{\tau}^{p}\right)^{2}\right]} \sim 1 \Rightarrow \alpha \sim v \sqrt{\frac{\epsilon_{c}}{K_{c}}} \tilde{F}_{c}
$$

where it is assumed that the velocity scales with $u_{\tau}^{p}$. We remark that the scaling factor $(1 / \alpha) \sqrt{K_{c} / \epsilon_{c}}$ in $(4.9 a)$ is now equal to $K_{c} /\left(\nu \epsilon_{c} \tilde{F}_{c}\right)$, which is the length scale associated with Forchheimer drag, see the last term in (4.6a). Thus, $\alpha$ has the expected limit for large $R e_{K}$.

\section{Numerical method}

In $\S 3$, the VANS equations were given for the homogeneous porous region, the interface region and the homogeneous fluid region. In a general and dimensionless form they read:

$$
\begin{aligned}
\frac{\partial \boldsymbol{u}}{\partial t}= & -\frac{1}{\epsilon} \nabla \cdot[\epsilon \boldsymbol{u} \boldsymbol{u}]-\nabla p+\frac{1}{R e_{b}} \nabla^{2} \boldsymbol{u}+\frac{1}{\epsilon R e_{b}} \nabla \epsilon \cdot \nabla \boldsymbol{u} \\
& -\frac{1}{R e_{b}} \frac{F o}{D a} \epsilon|\boldsymbol{u}| \boldsymbol{u}+\frac{1}{R e_{b}}\left[\frac{\nabla^{2} \epsilon}{\epsilon}-\frac{\epsilon}{D a}\right] \boldsymbol{u}, \\
\nabla \cdot[\epsilon \boldsymbol{u}]= & 0,
\end{aligned}
$$

where $\operatorname{Re}_{b} \equiv U_{b} H / v$ is the bulk Reynolds number with $U_{b}$ the bulk velocity in the channel (between $z=0$ and $z=H$ ), $D a \equiv K / H^{2}$ the Darcy number, and $F o \equiv \tilde{F} U_{b}$ the Forchheimer number. The boundary conditions are the no-slip and no-penetration conditions at $z=-h$ and $z=H$.

To solve (5.1a) and (5.1b), we consider a channel with finite dimensions and use periodic boundary conditions in the wall-parallel directions. The equations are discretized in space on a Cartesian mesh, which is non-uniform and staggered in the $z$-direction. The mesh points are clustered around $z=0$ (permeable wall) and $z=H$ (top wall). The stretch factor, i.e. the difference in cell width between two neighbouring cells, is always less than $3.3 \%$. It is largest at $z=-h$ and $z=H / 2$, and it gradually becomes smaller near $z=0$ and $z=H$. We use a pseudospectral method for the spatial derivatives in the wall-parallel directions, and a finite-volume method with the central-differencing scheme for the wall-normal direction. The equations are advanced in time with a pressure-correction method. All terms, except the last one, on the right-hand side of (5.1a) are integrated in time with the second-order explicit Adams-Bashforth scheme. For the last term, the second-order implicit CrankNicolson scheme is used (Wesseling 2001). This avoids a small computational time step in case of small values of the Darcy number. Our scheme reads:

$$
\begin{aligned}
\frac{\hat{u_{i}}-u_{i}^{n}}{\Delta t} & =\frac{3}{2} R_{i}^{n}-\frac{1}{2} R_{i}^{n-1}-\frac{\mathrm{d} P}{\mathrm{~d} x} \delta_{i 1}+\frac{1}{2} S_{i}\left(\hat{u}_{i}+u_{i}^{n}\right), \\
\frac{\partial}{\partial x_{i}}\left(\epsilon \frac{\partial \hat{p}}{\partial x_{i}}\right) & =\frac{1}{\Delta t} \frac{\partial \epsilon \hat{u}_{i}}{\partial x_{i}} \\
u_{i}^{n+1} & =\hat{u}_{i}-\Delta t \frac{\partial \hat{p}}{\partial x_{i}}, \\
p^{n+1} & =\hat{p}+2 p^{n}-p^{n-1},
\end{aligned}
$$




$\begin{array}{llcccccccccr}\text { Case } & \epsilon_{c} & D a_{c} & F o_{c} & R e_{\tau}^{t} & \Delta x^{t+} & \Delta y^{t+} & \Delta z_{c}^{t+} & \Delta z_{p}^{t+} & \Delta t^{t+} & \Delta T_{b} & N \\ \text { E95 } & 0.95 & 1.9 \times 10^{-4} & 10.5 & 407 & 7.9 & 6.4 & 0.5-5.3 & 0.5-8.5 & 0.014 & 53 & 60 \\ \text { E80 } & 0.8 & 7.1 \times 10^{-6} & 2.2 & 354 & 6.9 & 5.5 & 0.4-4.6 & 0.4-7.4 & 0.015 & 65 & 100 \\ \text { E60 } & 0.6 & 7.5 \times 10^{-7} & 0.83 & 350 & 6.8 & 5.5 & 0.4-4.6 & 0.4-7.3 & 0.017 & 66 & 86 \\ \text { E0 } & 0 & 0 & 0 & 350 & 6.8 & 5.5 & 0.4-4.6 & - & 0.017 & 89 & 58\end{array}$

TABLE 1. Characteristics of the simulations. $R e_{\tau}^{t} \equiv u_{\tau}^{t} H / v . \Delta x^{t+}$ and $\Delta y^{t+}$ are the mesh spacings in, respectively, the streamwise and the spanwise direction, normalized by $v / u_{\tau}^{t} . \Delta z_{c}^{t+}$ and $\Delta z_{p}^{t+}$ are the dimensionless mesh spacings in the wall-normal direction in, respectively, the channel and the permeable wall. $\Delta t^{t+}$ is the time step, normalized by $v /\left(u_{\tau}^{t}\right)^{2}, \Delta T_{b}$ is the time interval over which the statistics are obtained in units of $H / U_{b}$, and $N$ is the number of instantaneous data fields used for the statistics.

where $\hat{u}_{i}$ and $\hat{p}$ are, respectively, the prediction velocity and the correction pressure, $\mathrm{d} P / \mathrm{d} x$ is the mean pressure gradient that drives the flow, and $R_{i}$ and $S_{i}$ are defined as:

$$
\begin{aligned}
R_{i} & \equiv-\frac{1}{\epsilon} \frac{\partial \epsilon u_{i} u_{j}}{\partial x_{j}}-\frac{\partial p}{\partial x_{i}}+\frac{1}{R e_{b}} \frac{\partial^{2} u_{i}}{\partial x_{j}^{2}}+\frac{1}{\epsilon e_{b}} \frac{\partial \epsilon}{\partial x_{j}} \frac{\partial u_{i}}{\partial x_{j}}-\frac{1}{R e_{b}} \frac{F o}{D a} \epsilon|\boldsymbol{u}| u_{i}, \\
S_{i} & \equiv \frac{1}{R e_{b}}\left(\frac{1}{\epsilon} \frac{\partial^{2} \epsilon}{\partial x_{j}^{2}}-\frac{\epsilon}{D a}\right) .
\end{aligned}
$$

The value of $\mathrm{d} P / \mathrm{d} x$ is adjusted in time to enforce a constant bulk velocity in the channel. In the Crank-Nicolson scheme, the velocity at time step $n+1$ is estimated by the prediction velocity, but it can be shown that our pressure correction scheme is still second-order accurate. The time step $\Delta t$ is calculated from the constraints for numerical stability as derived by Wesseling (2001) for the second-order AdamsBashforth scheme.

We will show results from four simulations, for which we only changed the porosity $\epsilon_{c}$ in the homogeneous porous region. We will refer to them as E95, E80, E60 and E0, with corresponding porosities of, respectively, 0.95, 0.8, 0.6 and 0 (solid wall). In all simulations the mean particle diameter is fixed at $d_{p} / H=0.01$. The thickness of the interface region is taken equal to $\delta_{i} / d_{p}=2$. The thickness of the permeable wall is $h / H=1$, and has been chosen deliberately large to avoid any influence of the solid wall at $z=-h$ on the flow in the channel. The horizontal dimensions of the computational domain are, respectively, $L_{x} / H=5$ and $L_{y} / H=3$. The number of mesh points is equal to $256 \times 192 \times(128+192)$ in, respectively, the streamwise, spanwise and wall-normal direction. The bulk Reynolds number is fixed at $R e_{b}=5500$. More characteristics are given in table 1 .

The initial conditions for the simulations were either a random velocity field with a prescribed mean velocity profile, or an instantaneous velocity field from a previous simulation. In each simulation, the time step was initially adapted to the largest value for which the criteria for numerical stability were satisfied. When the value of $\mathrm{d} P / \mathrm{d} x$ had reached a steady state, the time step was fixed in time at the value given in table 1 .

\section{Numerical results}

\subsection{Mean velocity}

Figure 3 shows the mean velocity profile for different values of the wall porosity $\epsilon_{c}$. The permeable wall in case E60 behaves apparently similarly to a solid wall, 

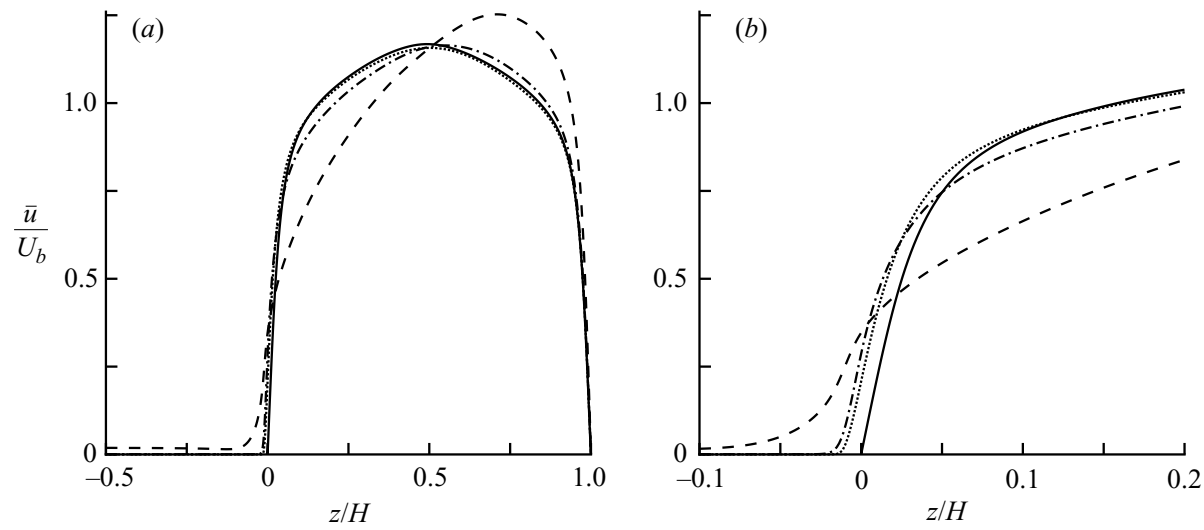

FiguRE 3. (a) Mean-velocity profile for different values of $\epsilon_{c}$, normalized by the bulk velocity $U_{b}$ and shown as a function of the dimensionless height $z / H$. For clarity, the region $-1 \leqslant z / H<-0.5$ is omitted because of a nearly uniform flow. (b) Enlargement of the region around the wall interface. - , case E0; $\cdots$, case E60; $-\cdot-$, case E80; -- - , case E95.

\begin{tabular}{lllllllllr}
\hline Case & $\epsilon_{c}$ & $R e_{\tau}^{p}$ & $R e_{K}$ & $R e_{d}$ & $\delta_{w} / H$ & $R e_{\delta}^{p}$ & $R e_{\delta}^{t}$ & $C_{f}^{t}\left(\times 10^{-3}\right)$ & $C_{f}^{p}\left(\times 10^{-3}\right)$ \\
E95 & 0.95 & 678 & 9.35 & 6.78 & 0.74 & 498 & 108 & 10.9 & 30.4 \\
E80 & 0.8 & 398 & 1.06 & 3.98 & 0.56 & 222 & 156 & 8.3 & 10.5 \\
E60 & 0.6 & 353 & 0.31 & 3.53 & 0.50 & 176 & 175 & 8.1 & 8.2 \\
E0 & 0 & 352 & 0 & 0 & 0.50 & 176 & 175 & 8.1 & 8.2
\end{tabular}

TABLE 2. Additional flow characteristics of the simulations. The superscripts $t$ and $p$ refer to, respectively, the top wall and the permeable wall region. $R e_{\tau}^{p} \equiv u_{\tau}^{p} H / v, R e_{K} \equiv \sqrt{K_{c}} u_{\tau}^{p} / v$, $R e_{d} \equiv d_{p} u_{\tau}^{p} / v, \delta_{w}$ is defined by (4.4), $R e_{\delta}^{p} \equiv \delta_{w} u_{\tau}^{p} / v, R e_{\delta}^{t} \equiv\left(H-\delta_{w}\right) u_{\tau}^{t} / v$ and $C_{f} \equiv 2\left(u_{\tau} / U_{b}\right)^{2}$.

because the velocity profiles of cases E60 and E0 overlap. For higher wall porosities, the velocity profile is more skewed, with its maximum $U_{m}$ located closer to the top wall. Based on (4.4), this implies that the skin friction coefficient $C_{f} \equiv 2\left(u_{\tau} / U_{b}\right)^{2}$ is larger for the permeable bottom wall than for the solid top wall. In case E95, the difference amounts to almost a factor of three, see table 2. The large skin friction at the permeable wall in case E95 is responsible for a significant increase in the ratio $U_{m} / U_{b}$ as compared to case E0, because by definition $(1 / H) \int_{0}^{H}\left(\bar{u} / U_{b}\right) \mathrm{d} z=1$ must hold. It even causes an increase of about $35 \%$ in the skin friction coefficient at the top wall.

From figure $3(b)$ it can be seen that the mean velocity profile exhibits a point of inflection $\left(\mathrm{d}^{2} \bar{u} / \mathrm{d} z^{2}=0\right)$ just below the interface with the permeable wall. According to Rayleigh's criterion (Drazin \& Reid 1981), a point of inflection in the velocity profile is a necessary condition for an inviscid instability, at least for the case of unidirectional laminar flow in a homogeneous fluid region. The velocity profile could therefore give rise to instabilities of the Kelvin-Helmholtz type, similar to the instabilities present in a plane mixing layer, as has been suggested before by Raupach, Antonia \& Rajagopalan (1991) and Finnigan (2000) for flows over plant canopies.

Figure 4 shows a two-dimensional snapshot of an instantaneous flow field in case E95. Near the permeable wall, relatively large vortical structures are present, which seems to substantiate the hypothesis of a Kelvin-Helmholtz type of instability. Notice furthermore that the turbulence structure is clearly very different in the boundary 


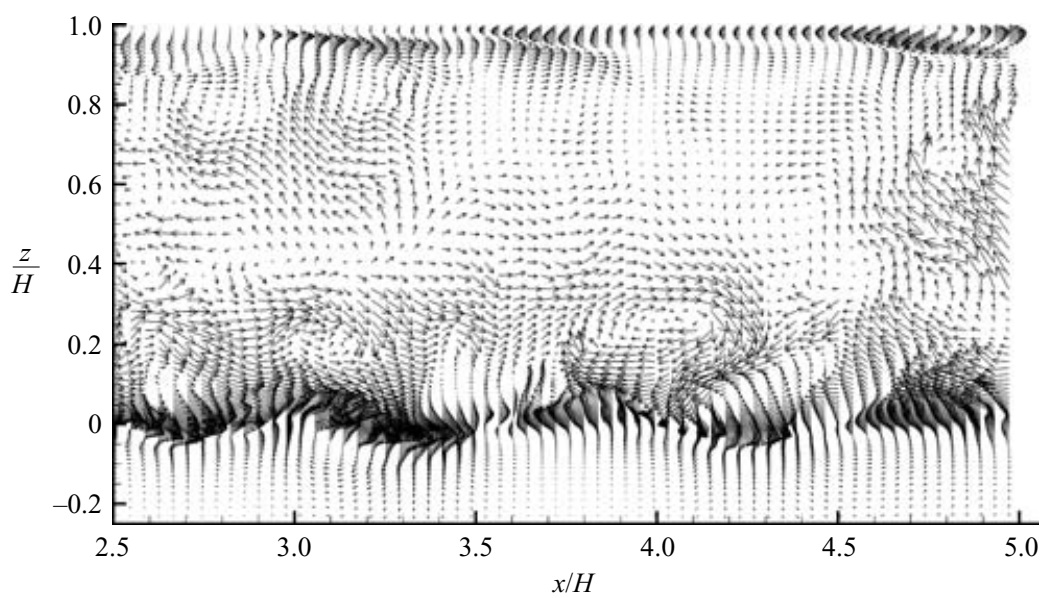

FIGURE 4. Two-dimensional snapshot of an instantaneous flow field $\left(u^{\prime}, w^{\prime}\right)$ in case E95. Note that the lower part of the permeable wall is not shown, because velocities in this region are much smaller than in the channel region.
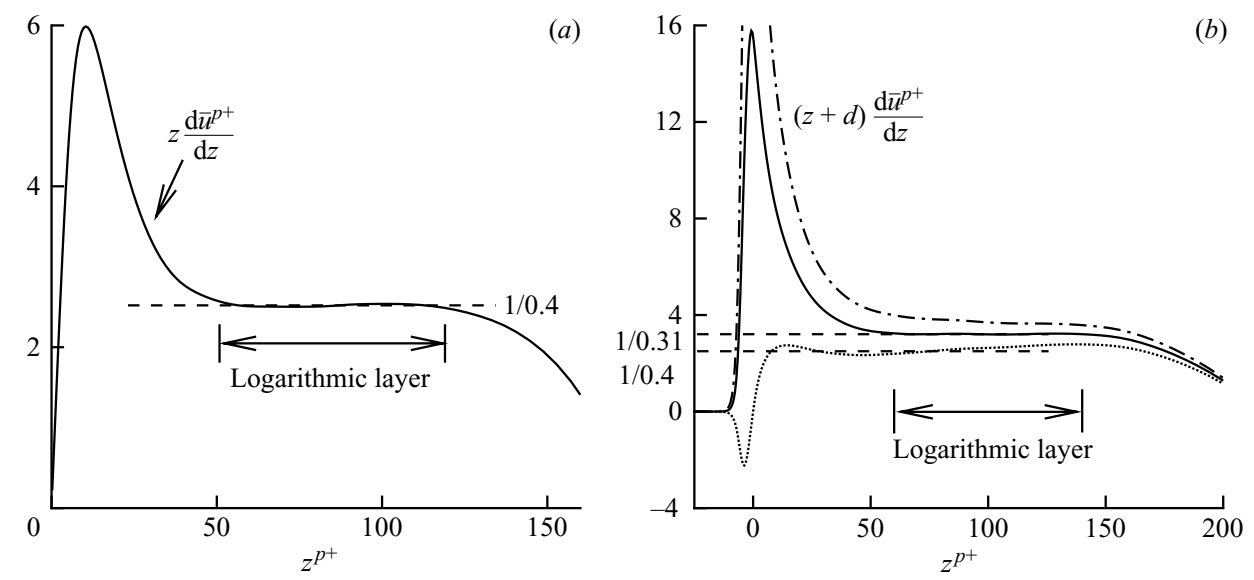

FIGURE 5. $(a)(z+d) \mathrm{d} \bar{u}^{p+} / \mathrm{d} z$ as function of $z^{p+} \equiv z u_{\tau}^{p} / v$ for case E0 with $d^{p+}=0$. (b) The same but for case E80. The lines correspond to different values of $d^{p+}: \cdots, d^{p+}=0$; $d^{p+}=21 ;-\cdot-, d^{p+}=40$. Note that the lines are plotted as function of $z^{p+}$, rather than $(z+d)^{p+}$, because of the different values for $d$.

layer above the permeable wall compared to the boundary layer below the top wall. Near the top wall, elongated streaky structures are observed, which are absent near the permeable wall.

To investigate the properties of the mean flow, the log law (4.5) has been fitted to the mean velocity profile. First the extent of the logarithmic layer is determined from a plot of $(z+d) \mathrm{d} \bar{u}^{p+} / \mathrm{d} z$ as a function of $z^{p+} \equiv z u_{\tau}^{p} / v$ for several values of $d^{p+}$. Inside the logarithmic layer this quantity must be a constant equal to $1 / \lambda$. Plots of $(z+d) \mathrm{d} \bar{u}^{p+} / \mathrm{d} z$ are shown in figure 5 for cases E0 and E80. In case E0, $d=0$, because the channel walls are impermeable. A logarithmic layer is present for approximately $z^{p+} \in[50: 120]$. The value of $\lambda$ is equal to 0.40 , which is in agreement with the value of approximately 0.4 for the von Kármán constant for flows over smooth walls. In case E80, a logarithmic layer is found in the range $z^{p+} \in[60: 140]$ when $d^{p+}$ is taken 


$\begin{array}{lccccccccccc}\text { Case } & \lambda & z_{0} / \sqrt{K_{c}} & z_{0} / d_{p} & z_{0} / \delta_{w} & z_{0}^{p+} & d / \sqrt{K_{c}} & d / d_{p} & d / \delta_{w} & d^{p+} & d^{p+}(\mathrm{JM}) & \alpha \\ \text { E95 } & 0.23 & 5.30 & 7.32 & 0.10 & 49.6 & 11.3 & 15.6 & 0.21 & 105.6 & 16.7 & 0.70 \\ \text { E80 } & 0.31 & 1.33 & 0.35 & 0.006 & 1.41 & 20.0 & 5.3 & 0.10 & 21.2 & 4.7 & 0.99 \\ \text { E60 } & 0.40 & 0.35 & 0.03 & 0.0006 & 0.11 & 0 & 0 & 0 & 0 & 3.4 & - \\ \text { I } & 0.40 & \infty & \infty & 0.0006 & 0.11 & 0 & 0 & 0 & 0 & 0 & -\end{array}$

TABLE 3. Fit parameters. $\lambda$ is equivalent to the von Kármán constant for a solid wall. $d$ and $z_{0}$ are the length scales in the log law (4.5), normalized by, respectively, $\sqrt{K_{c}}, d_{p}, \delta_{w}$ and $v / u_{\tau}^{p}$. The value of $d^{p+}$ in the penultimate column is obtained from Jackson's model (JM), equation (6.1). $\alpha$ is obtained from a least-squares fit of (4.5) to the mean velocity profile in the region $z / H \in[-0.85:-0.02]$.
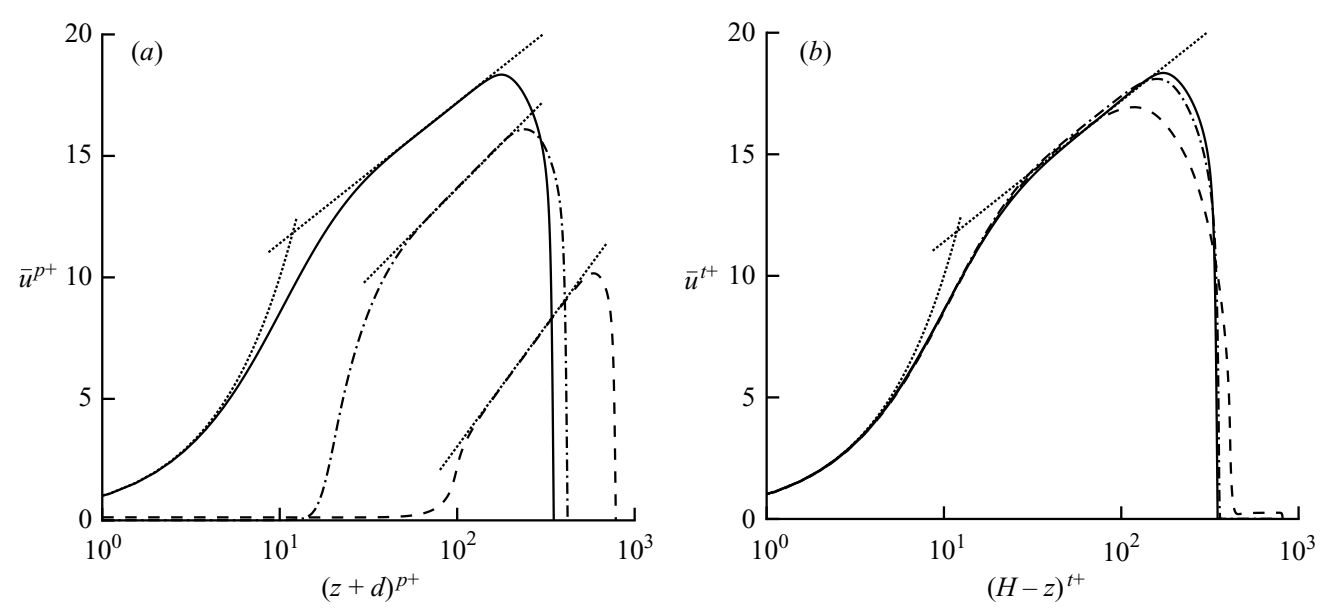

FiguRE 6. (a) Mean velocity $\bar{u}^{p+}$ in the region above the permeable wall for different bottom wall porosities, shown as a function of the dimensionless shifted height $(z+d)^{p+}$. (b) The same, but now in the region below the top wall with $\bar{u}^{t+} \equiv \bar{u} / u_{\tau}^{t}$ and $(H-z)^{t+}$ the distance to the top wall. - , case E0; -.-, case E80; ---, case E95. The dotted straight lines are least-square fits of the logarithmic law to the velocity profiles. The fit parameters are given in table 3 . The dotted curved lines correspond to $\bar{u}^{p+}=z^{p+}$ and $\bar{u}^{t+}=(H-z)^{t+}$, respectively.

equal to 21 . The corresponding value of $\lambda$ is 0.31 and this is significantly lower than 0.4. We emphasize that it is not possible to choose $d^{p+}$ such that $\lambda$ is close to 0.4 , which is clear from figure $5(b)$. In case E95, $\lambda$ is even found to be 0.23 . A similar low value for $\lambda$ has also been found for the DNS of Breugem \& Boersma (2005) of channel flow over a grid of cubes, although not discussed in their paper. These values are slightly dependent of the choice of the fit interval, but the variation in $\lambda$ is small. In case E60, the value of $d^{p+}$ was too small to obtain a conclusive value, and therefore it was taken as zero. The corresponding value of $\lambda$ is equal to 0.40 , the same as in case E0. The values of $d, z_{0}$ and $\lambda$ obtained from a least-squares fit of (4.5) to the velocity profile in the logarithmic layer, are given in table 3. A lin-log plot of the mean velocity in the boundary layer above the permeable wall, along with the logarithmic fits, is shown in figure $6(a)$. Figure $6(b)$ shows a lin-log plot of the mean velocity near the solid top wall. The profiles coincide, which indicates that the scaling of the mean flow near the top wall is not altered by the presence of the permeable bottom wall. 


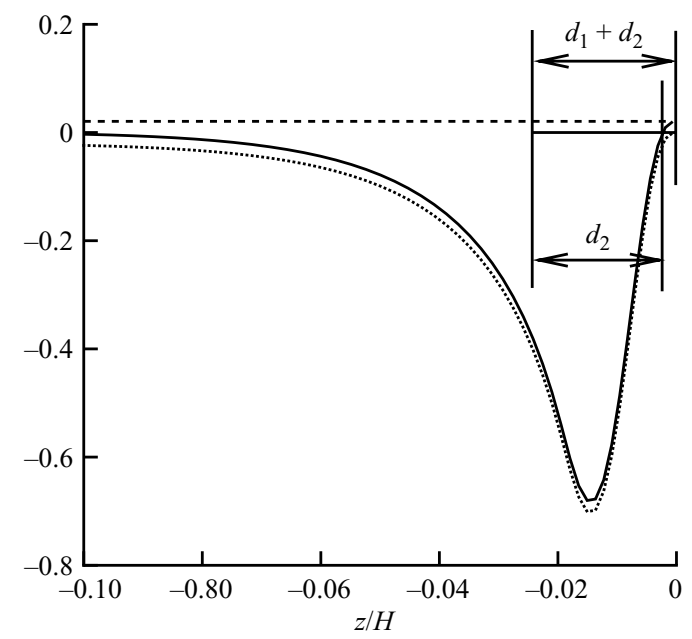

FIGURE 7. Profiles of the pressure gradient, the drag force and the effective drag force in case E95, normalized by $\rho U_{b}^{2} / H$ and shown as functions of $z / H . \cdots, f ;---,-\mathrm{d} \bar{p} / \mathrm{d} x ;-f_{e} . d_{1}$ and $d_{2}$ are contributions to the displacement height based on Jackson's model.

For both smooth and rough walls, $\lambda$ is usually found to be approximately 0.4 (Raupach et al. 1991). The value of $\lambda$ in cases E80 and E95 is significantly smaller than this. Also the ratio of $z_{0} / d_{p}$ in cases E80 and E95 is much larger than the value of approximately $1 / 30$ found from Nikuradse's experiments of flows over fully rough walls covered with homogeneous sand roughness (Yaglom 1979, p. 513). According to Hinze (1975; p. 637), for fully rough walls, the displacement height in the log law is found to scale with the mean particle diameter $d_{p}$ according to $d / d_{p} \approx 0.3$. For case $\mathrm{E} 95, d / d_{p}=15.6$ and for case $\mathrm{E} 80, d / d_{p}=5.3$, see table 3, which is much larger than 0.3 . Thus, the permeable walls in cases E80 and E95 behave very differently from fully rough walls. We note that according to the classification of Hinze (1975), the effect of surface roughness is negligible in these cases, because in case E80, the roughness Reynolds number $R e_{d}=4$ and in case $\mathrm{E} 95, R e_{d}=6.8$, see table 2 . The unusual values for $\lambda, d$ and $z_{0}$ are apparently related to the permeability of the wall.

For boundary-layer flow over a porous wall in the absence of a mean pressure gradient, Jackson (1981) proposed that the displacement height $d$ in the log law is related to the level inside the wall at which the height-averaged drag force acts. Consistent with this idea, we argue in Appendix B that in the presence of a mean pressure gradient, $d$ could then be calculated from:

$$
d=d_{1}+d_{2}, d_{2}=-\frac{\int\left(z+d_{1}\right) f_{e} \mathrm{~d} z}{\int f_{e} \mathrm{~d} z} \text { with } z<-d_{1},
$$

where $f_{e}$ is the effective drag force (i.e. drag force minus mean pressure gradient), and where $z=-d_{1}$ is the level at which $f_{e}=0$. Figure 7 presents profiles of $f,-\mathrm{d} \bar{p} / \mathrm{d} x$ and $f_{e}$ in case E95. Also indicated are $d_{1}$ and $d_{2}$ calculated from (6.1). Their sum is equal to $d^{p+}=16.7$ which is more than six times smaller than the value obtained from a $\log$ fit to the velocity profile. For all simulations, the values for $d$ calculated from (6.1) are given in table 3. Although the trend is correct, i.e. $d$ increases with increasing permeability, the modified Jackson model strongly underpredicts $d$. The 

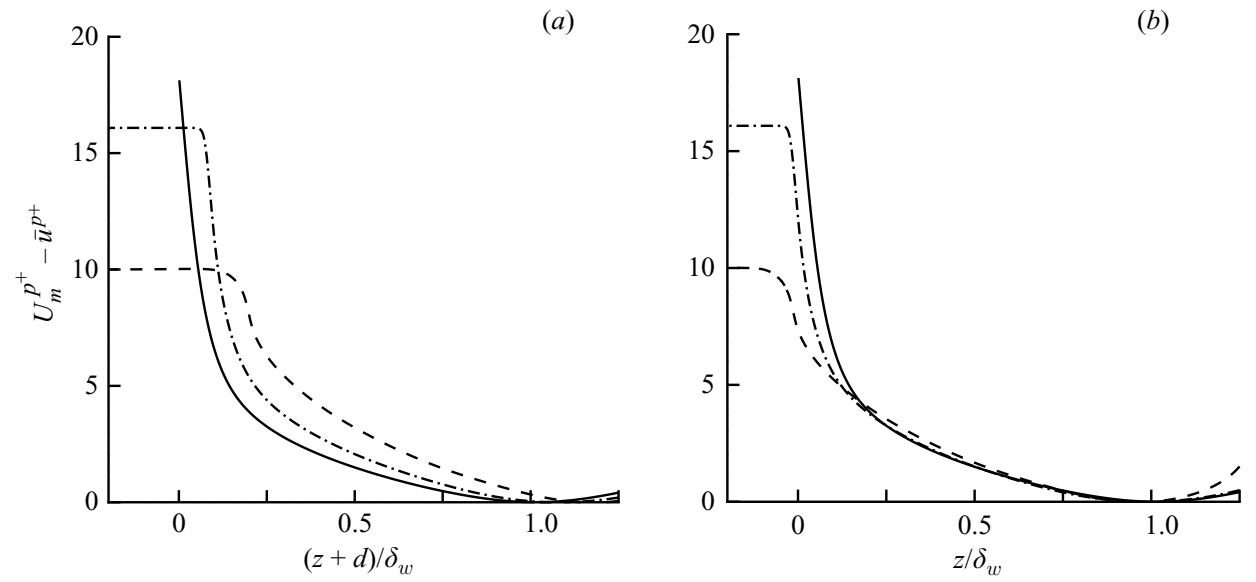

FIGURE 8. Velocity defect $U_{m}^{p+}-\bar{u}^{p+}$ for different wall porosities, plotted as functions of (a) $(z+d) / \delta_{w} ;(b) z / \delta_{w} .-$, case E0;-.-, case E80; - - , case E95.

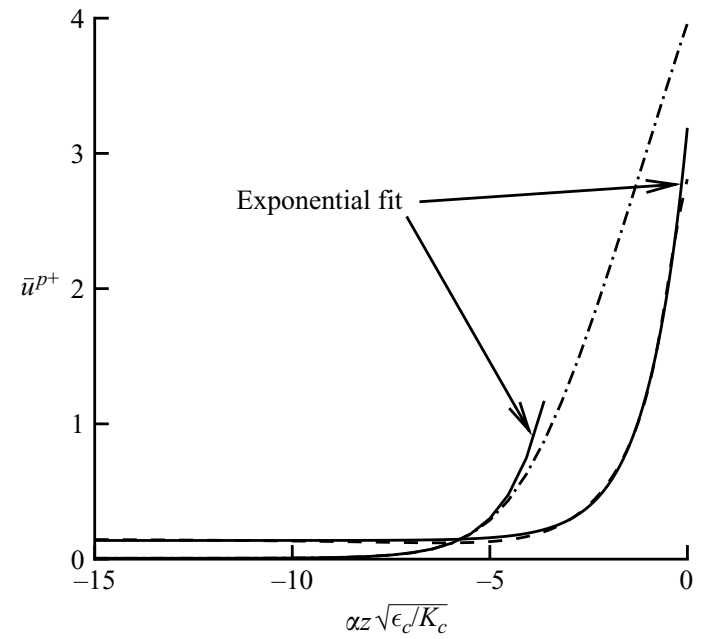

Figure 9. Mean velocity $\bar{u}^{p+}$ in the top region of the permeable wall as a function of the dimensionless height $\alpha z \sqrt{\epsilon_{c} / K_{c}} \cdot-\cdot-$, case E80; ---, case E95. The solid lines are fits of the exponential function (4.9a) to the velocity profiles. The coefficient $\alpha$ is given in table 3 .

assumption that $z=-d$ is the level at which the height-averaged effective drag force acts, is apparently not correct for the present simulations.

Figure $8(a)$ shows the velocity defect $U_{m}^{p+}-\bar{u}^{p+}$ as a function of $(z+d) / \delta_{w}$. It is observed that the velocity defect does not scale with $(z+d) / \delta_{w}$, as is the case for flows over a rough wall at sufficiently high Reynolds number. Figure $8(b)$ shows instead that the velocity defect seems to scale with $z / \delta_{w}$ for $z / \delta_{w} \gtrsim 0.2$. This observation suggests that the large eddies in the outer region are blocked by the permeable wall, or sheltered by the mean-shear layer at the interface (Hunt \& Durbin 1999).

We will now investigate the velocity profile inside the permeable wall. As discussed in $\S 4$, the mean flow is expected to decrease exponentially inside the permeable wall. This is confirmed by figure 9 , which shows the mean velocity in the top region of the permeable wall as a function of $\alpha z \sqrt{\epsilon_{c} / K_{c}}$. The value of $\alpha$ is obtained from a 
least-squares fit of $(4.9 a)$ to the velocity profile in the homogeneous porous region. In case E60, $\alpha$ could not be determined, because at $z / H=-0.02$ the mean velocity has already reached the Darcy velocity $U_{d}$. In case E80, the value of $\alpha$ is 0.99 . This indicates that in the homogeneous porous region, the mean flow is governed by the Brinkman equations with the corresponding solution given by $(4.8 a)$. In case E95, $\alpha \approx 0.70$, which suggests that turbulent diffusion dominates over viscous diffusion in the top layer of the permeable wall. Notice that the exponential solution (4.9a) gives not only a good fit in the homogeneous porous region, but also in the interface region up to $z=0$.

\subsection{Mean kinetic energy}

To gain insight into the dynamics of the mean flow, the terms in the transport equation for the mean kinetic energy (MKE) $\bar{u}^{2} / 2$ have been investigated. This equation is obtained from multiplying the equation for the mean velocity by $\bar{u}$ :

$$
\begin{aligned}
0= & \underbrace{-\bar{u} \frac{\mathrm{d} \bar{p}}{\mathrm{~d} x}}_{P}+\underbrace{\overline{u^{\prime} w^{\prime}} \frac{\partial \bar{u}}{\partial z}}_{W S}-\underbrace{-\frac{1}{\epsilon} \frac{\partial \epsilon \bar{u} \overline{u^{\prime} w^{\prime}}}{\partial z}}_{T T}+\underbrace{\frac{1}{R e_{b}} \frac{\partial^{2} \frac{1}{2} \bar{u}^{2}}{\partial z^{2}}}_{V D} \underbrace{-\frac{1}{R e_{b}}\left(\frac{\partial \bar{u}}{\partial z}\right)^{2}}_{D} \\
& +\underbrace{\frac{1}{R e_{b}} \frac{1}{\epsilon} \frac{\partial \epsilon}{\partial z} \frac{\partial \frac{1}{2} \bar{u}^{2}}{\partial z}+\frac{1}{R e_{b}} \frac{1}{\epsilon} \frac{\partial^{2} \epsilon}{\partial z^{2}} \bar{u}^{2}}_{P O R} \underbrace{-\frac{1}{R e_{b}} \frac{\epsilon}{D a} \bar{u}^{2}}_{D A R} \underbrace{-\frac{1}{R e_{b}} \frac{\epsilon F O}{D a} \overline{|\boldsymbol{u}| u} \bar{u}}_{F O R} .
\end{aligned}
$$

Term $P$ represents the work by the mean pressure gradient, term $W S$ represents the loss due to deformation work by the Reynolds-shear stress, terms TT and $V D$ represent, respectively, turbulent transport and viscous diffusion, and term $D$ represents viscous dissipation. The last three terms on the right-hand side are only non-zero inside the permeable wall. The $P O R$-terms originate partly from viscous diffusion and partly from viscous drag, and represent transport and dissipation of energy by local changes in the porosity. The DAR- and the FOR-terms represent the transfer of resolved-scale MKE, i.e. the MKE of the volume-averaged flow, to subfilter-scale MKE by Darcy drag and Forchheimer drag, respectively. The terms in (6.2) are plotted in figure 10 for cases E95, E80 and E0. The ultimate source of MKE is the work by the mean pressure gradient $-\bar{u} \mathrm{~d} \bar{p} / \mathrm{d} x$, and that is why we have chosen to normalize the budget terms by $U_{m}\left[\left(u_{\tau}^{p}\right)^{2}+\left(u_{\tau}^{t}\right)^{2}\right] / H$. For clarity, the terms $P O R$, $D A R$ and FOR are shown separately from the other terms. The sum of all computed terms in (6.2) is very small compared to the dominant terms.

It is observed from figure $10(b, d, e)$ that sufficiently far away from the permeable wall, term $P$ is in local balance with terms $W S$ and $T T$. Term $T T$ is responsible for transport of MKE from the channel towards the permeable wall. Close to the wall, there are considerable differences between the cases. In case E95, terms $V D$ and $D$ are almost negligible. Term $T T$ peaks just below the wall interface, balancing terms $W S$ and FOR. This observation confirms our suggestion in the previous section, based on the value of $\alpha \approx 0.7$, that in case E95, turbulent diffusion dominates over viscous diffusion in the top layer of the permeable wall. In the core of the permeable wall $\left(z / \delta_{w} \lesssim-0.15\right)$, term $P$ balances terms $D A R$ and FOR.

In case E80, term $T T$ peaks just above the permeable wall and vanishes just below the interface, in a region where viscous diffusion is balanced by viscous dissipation. Further down in the porous medium, term $P$ balances term $D A R$. These observations show that in case E80 the mean flow inside the permeable wall is governed by the Brinkman equations, as suggested in the previous section based on the value of $\alpha \approx 1$. 

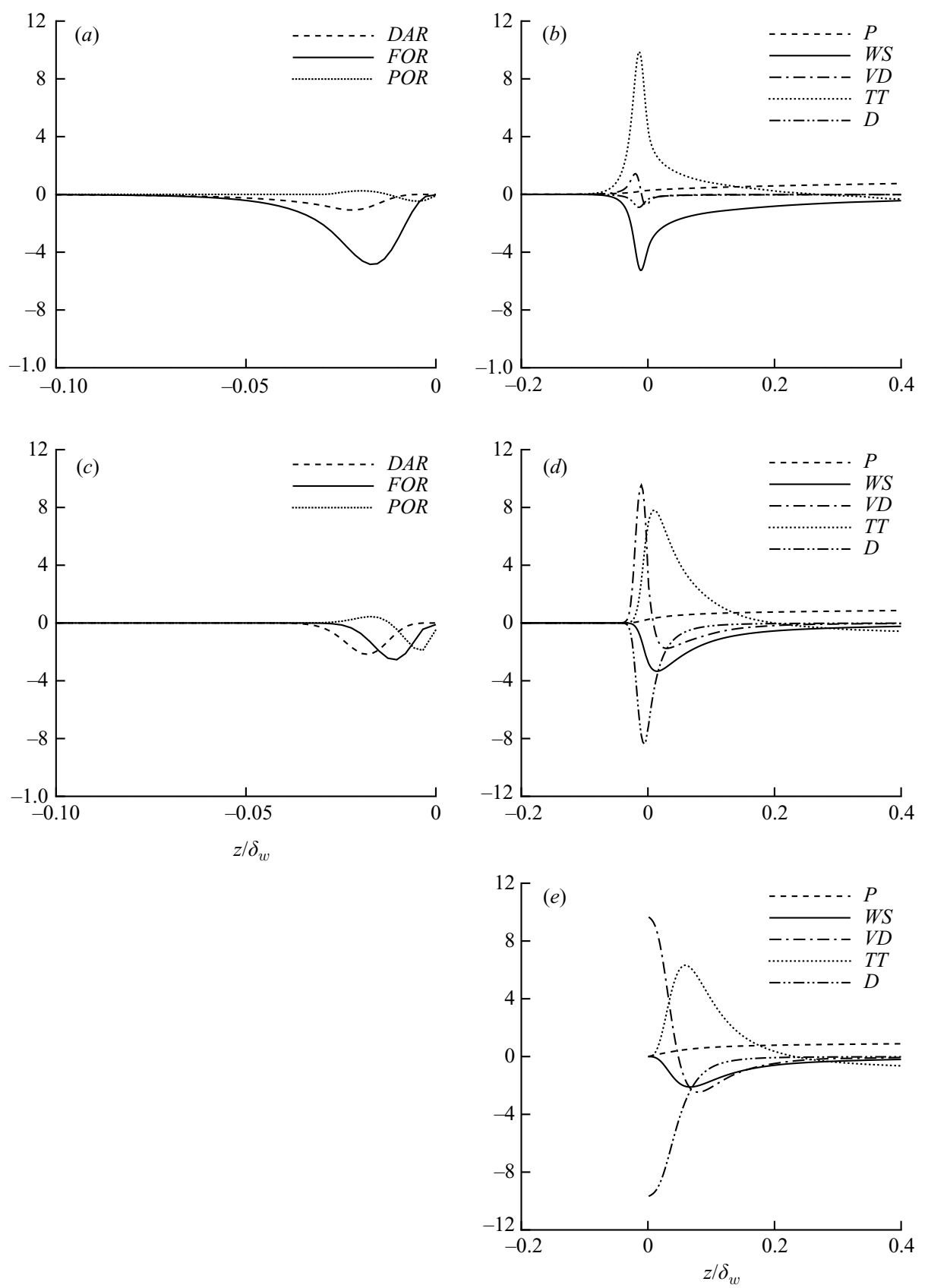

FiguRE 10. Terms in the transport equation for the mean kinetic energy $\bar{u}^{2} / 2$ for different wall porosities, normalized by $U_{m}\left[\left(u_{\tau}^{p}\right)^{2}+\left(u_{\tau}^{t}\right)^{2}\right] / H$ and shown as function of $z / \delta_{w} .(a, b)$ case E95; $(c, d)$ case E80; $(e)$ case E0.

Comparing cases E95, E80 and E0, we observe that in case E95, viscous effects are almost negligible and that in case E80, viscous effects are more confined to the permeable wall relative to case E0. Similar to the classification of rough walls, where a fully rough wall is defined as a wall where the thickness of the viscous sublayer 
is much less than the roughness height, we give the following classification of the permeable walls in cases E95, E80 and E60.

(i) The permeable wall in case E95 is highly permeable.

(ii) The permeable wall in case E80 is partially permeable.

(iii) The permeable wall in case E60 is effectively impermeable.

As discussed in $\S 4$, whether or not a wall is permeable for the turbulent flow above the wall is basically determined by the permeability Reynolds number $R e_{K} \equiv \sqrt{K_{c}} u_{\tau}^{p} / \nu$. The values of $R e_{K}$ for the different simulations are given in table 2. In case E95, $R e_{K}=9.35$, which is apparently in the highly permeable regime, whereas a value of 0.31, as in case E60, corresponds to an effectively impermeable wall.

\subsection{Velocity and pressure fluctuations}

Figure 11 presents the profiles of the r.m.s. velocities for different values of the wall porosity $\epsilon_{c}$. The r.m.s. velocities are normalized by the friction velocity $u_{\tau}^{t}$ at the top wall and plotted as a function of $z / H$. The symbols represent the profiles from the DNS of Kim, Moin \& Moser (1987) of turbulent flow between two solid walls, which are in excellent agreement with the profiles of case E0. Close to the top wall the profiles coincide, as expected, which indicates that the influence of the permeable wall on the r.m.s. velocities near the top wall is negligible. In the boundary layer above the permeable wall, a strong increase is observed in the r.m.s. velocities with respect to the boundary layer below the top wall. This increase is most pronounced for the spanwise and wall-normal fluctuations, which can be attributed to weakening of the wall-blocking and wall-induced viscous effects (Perot \& Moin 1995) near a permeable wall relative to an impermeable wall. Note that the r.m.s. velocities vanish rapidly inside the permeable wall. This indicates that the turbulent flow in the channel is not influenced by the presence of the solid wall at $z / H=-1$, as we had assumed.

Figure 12 shows again the r.m.s. profiles of the velocity components, but now normalized by the friction velocity $u_{\tau}^{p}$ at the permeable wall and plotted as function of $z / \delta_{w}$. The profiles of the streamwise r.m.s. velocity coincide for $z / \delta_{w} \gtrsim 0.4$. This observation seems to substantiate the wall similarity hypothesis (Raupach et al. 1991), which states that for large Reynolds numbers, turbulence in the outer region of a boundary layer is unaffected by the geometrical properties of the wall. The profiles of the spanwise and the wall-normal r.m.s. velocities however, exhibit, less similarity. Similar observations were made in the experiments of Krogstad, Antonia \& Browne (1992) and Kro̊gstad \& Antonia (1999) on boundary-layer flows over rough walls. They reported a significant increase in the wall-normal r.m.s. velocity, which was attributed to a weakening of the wall-blocking effect, whereas the streamwise r.m.s. velocity remained unchanged as compared to a smooth wall.

Close to the permeable wall, the peak in the streamwise r.m.s. profile is lower for higher wall porosity, whereas the peaks in the profiles of the two other r.m.s. velocities are higher for higher wall porosity. The smaller peak in the streamwise r.m.s. profile is consistent with the absence of streaky structures above a highly permeable wall (see figure 4). The peak in the turbulent kinetic energy $\left(u_{r m s}^{2}+v_{r m s}^{2}+w_{r m s}^{2}\right) / 2$ therefore decreases for higher wall porosity, as can be observed from figure $12(d)$.

The r.m.s. velocities decrease rapidly when moving downwards inside the permeable wall, which is a consequence of drag. Deep in the permeable wall, the velocity fluctuations are governed by Darcy's law with the linearized Forchheimer correction:

$$
0=-\frac{\partial p^{\prime}}{\partial x_{i}}-\frac{\epsilon_{c}}{R e_{b} D a_{c}} u_{i}^{\prime}-\frac{\epsilon_{c}}{R e_{b} D a_{c}} F o_{c}\left[\bar{u} u_{i}^{\prime}+u^{\prime} \overline{u_{i}}\right] .
$$



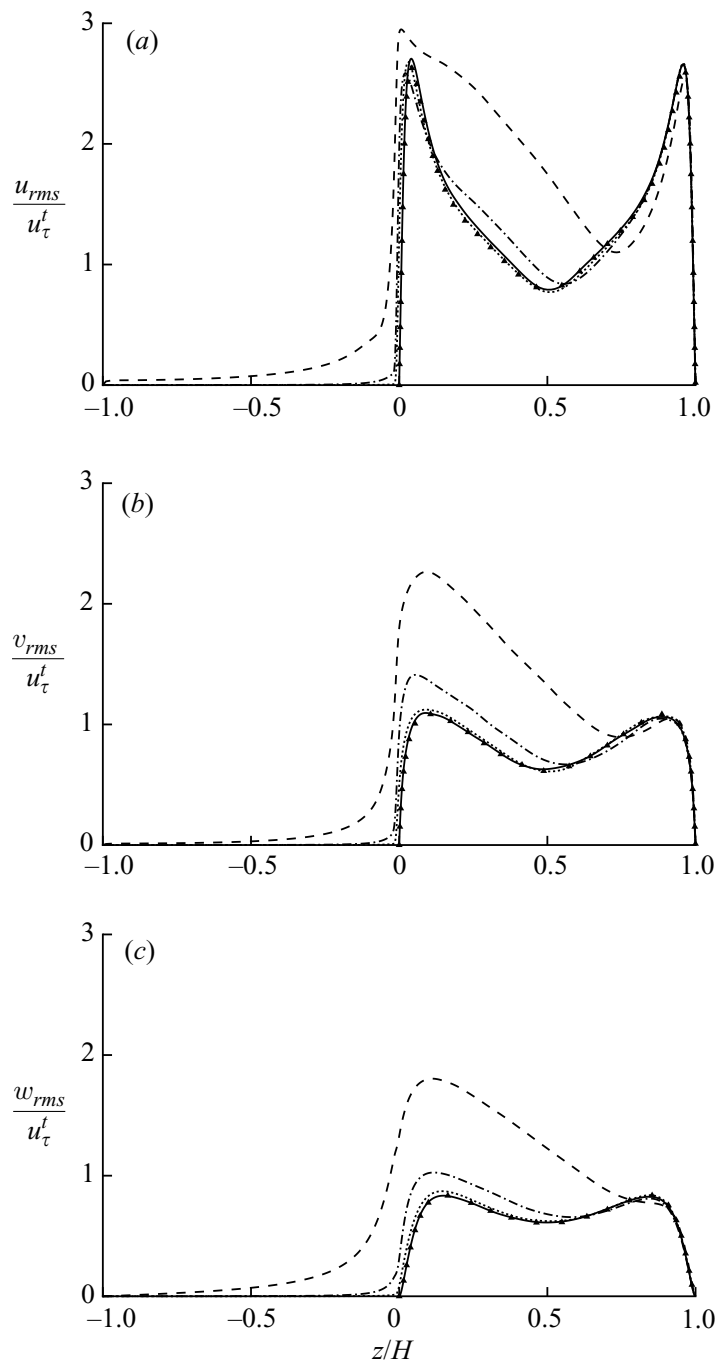

FIGURE 11. Profiles of the r.m.s. velocities, normalized by the friction velocity $u_{\tau}^{t}$ at the top wall, as function of $z / H$. The symbols refer to the DNS data of Kim et al. (1987). (a) streamwise component; (b) spanwise component; (c) wall-normal component. -, case E0; $\cdots$, case E60; ---, case E80; ---, case E95.

The Forchheimer correction is negligible in case E80, but not in case E95, although in that case, the Darcy term dominates far down in the permeable wall. Taking the divergence of this equation and assuming that $\partial \bar{u} / \partial z \approx 0$, we obtain a Laplace equation for the pressure fluctuations inside the permeable wall:

$$
\frac{\partial^{2} p_{i}^{\prime}}{\partial x_{i}^{2}} \approx 0
$$

The solution of this equation reads:

$$
p^{\prime}(x, y, z) \propto \sum_{k_{x}, k_{y}} A\left(k_{x}, k_{y}\right) e^{k z} \exp \left(\mathrm{i}\left(k_{x} x+k_{y} y\right)\right), \quad z<0,
$$



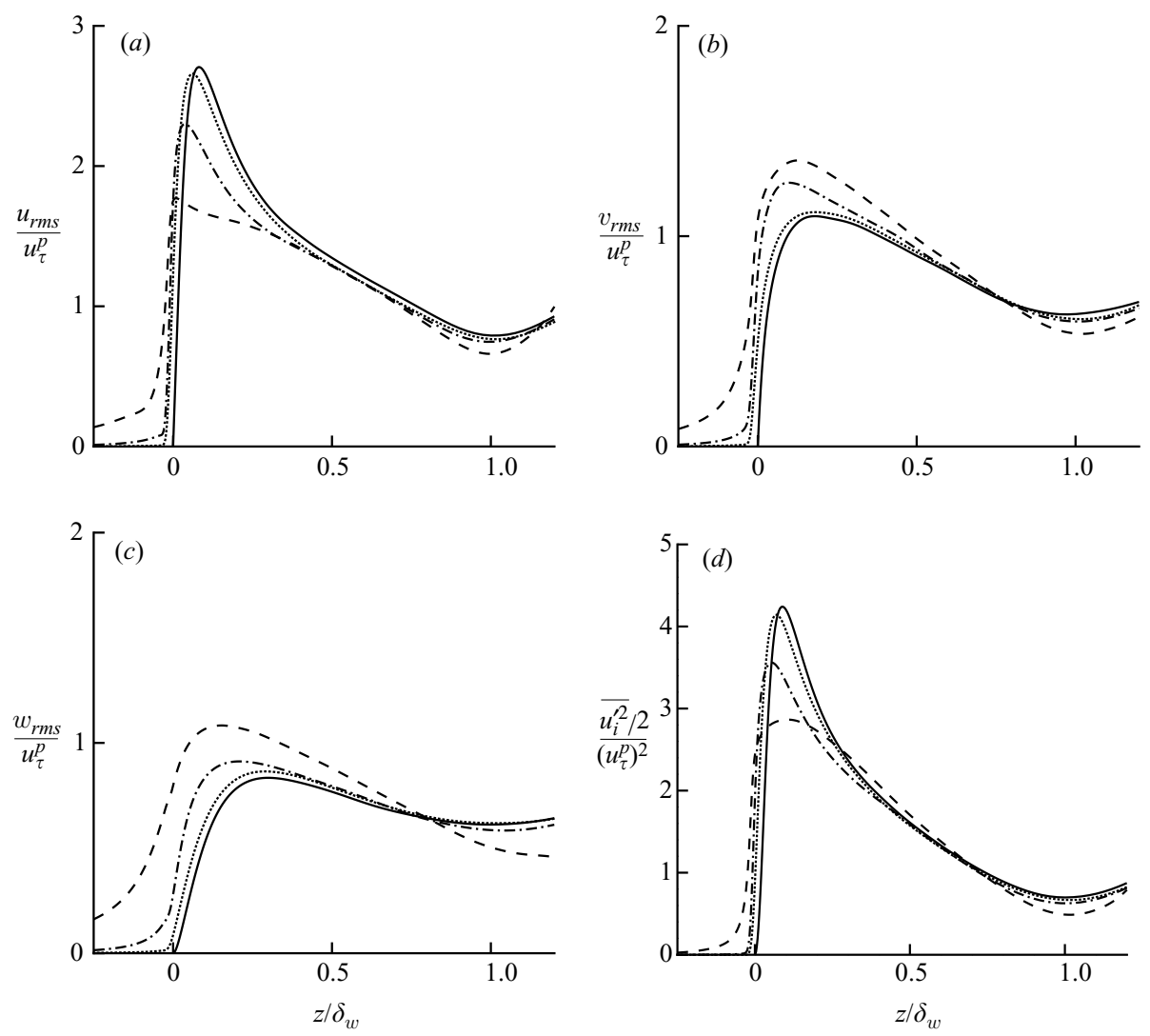

FIGURE 12. Profiles of the r.m.s. velocities and the turbulent kinetic energy, normalized by respectively $u_{\tau}^{p}$ and $\left(u_{\tau}^{p}\right)^{2}$, and plotted as function of $z / \delta_{w}$. (a) r.m.s. streamwise velocity; (b) r.m.s. spanwise velocity; $(c)$ r.m.s. wall-normal velocity; $(d)$ turbulent kinetic energy. case $\mathrm{E} 0 ; \cdots$, case E60;-.--, case E80; ---, case E95.

where $\mathrm{i}=\sqrt{-1}, k_{x}=2 \pi n_{x} / L_{x}$ and $k_{y}=2 \pi n_{y} / L_{y}$ are the wavenumbers in, respectively, the streamwise and spanwise directions with $n_{x}$ and $n_{y}$ integer numbers, $k=\left(k_{x}^{2}+k_{y}^{2}\right)^{1 / 2}$, and $A$ is a function of $k_{x}$ and $k_{y}$. The value of $A$ is related to the amplitude of the pressure fluctuations at the interface between the permeable wall and the channel. This solution predicts that the wave components of the fluctuations decrease exponentially, and that small-scale fluctuations decrease more rapidly than large-scale fluctuations. This behaviour is also observed in the streamwise pressure spectra shown in figure 13(a). Substitution of (6.5) into (6.3) shows that the wave components of the velocity components must also decrease exponentially when moving down in the permeable wall. This is confirmed by the streamwise spectra of the wall-normal velocity in figure $13(b)$ and it explains the exponential-like tail of the r.m.s. profiles inside the permeable wall (see figure 11).

Figure 14 presents the r.m.s. profiles of the pressure. The profiles of case E80 and case E0 coincide for $z \gtrsim 0.3$, which substantiates the wall similarity hypothesis. However, in the same range, the profile of case E95 lies significantly above the other profiles. The peak near the permeable wall is increasing for higher wall porosity. This can be related to the weakening of the wall-blocking effect; more details can be found in Breugem (2004). Inside the permeable wall the r.m.s. profiles exhibit an 

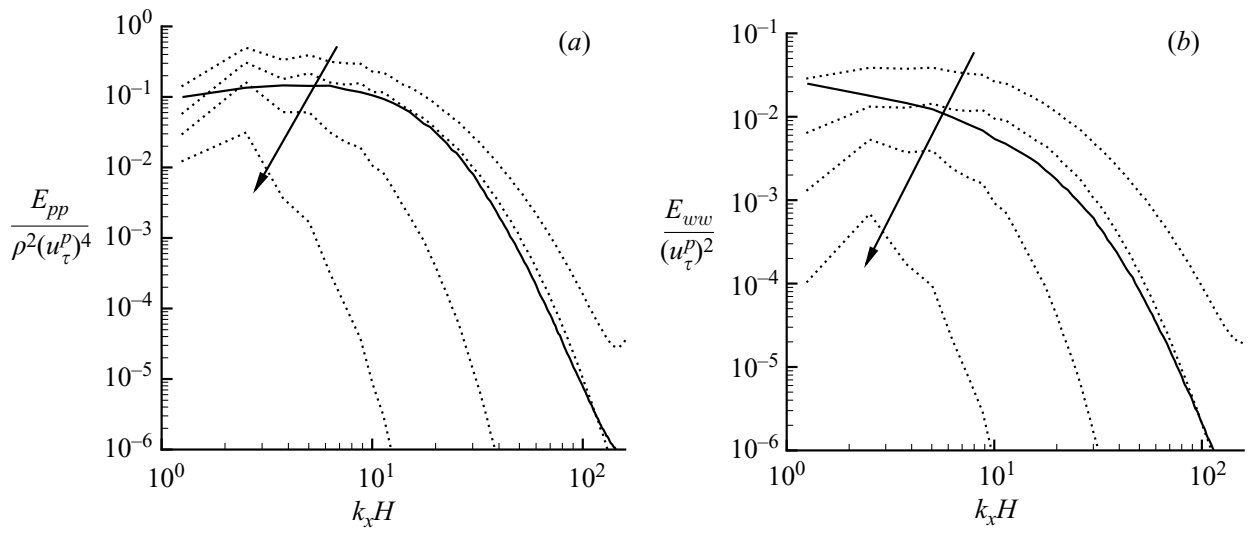

FIGURE 13. One-dimensional streamwise spectra of the pressure and the wall-normal velocity in case E95 (dotted lines) and case E0 (solid line). Each line corresponds to a different height, which is decreasing in the direction of the arrow: $z / H=-0.47,-0.14,-0.035,0.05$. The solid line of case E0 corresponds to $z / H=0.05$. (a) $E_{p p} ;(b) E_{w w}$.

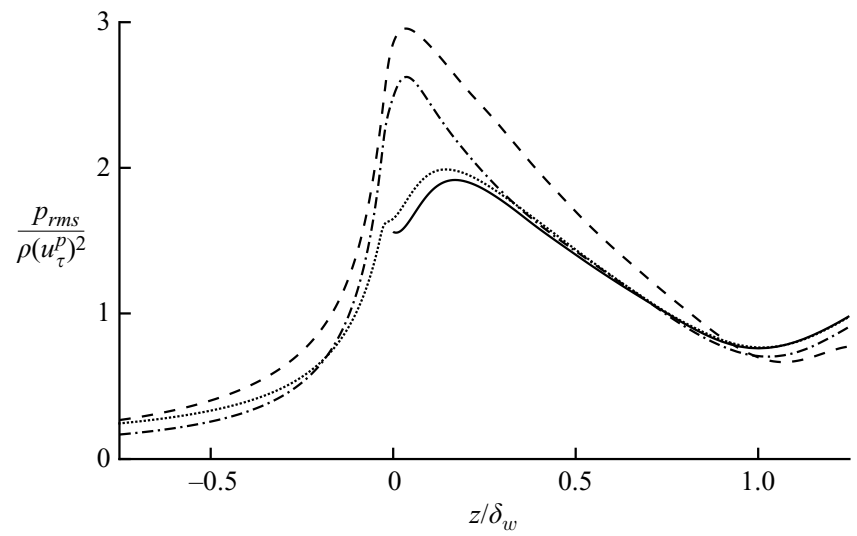

FIGURE 14. Root mean square profile of the pressure for different wall porosities, normalized by $\rho\left(u_{\tau}^{p}\right)^{2}$, as a function of $z / \delta_{w} \cdot-$, case E0; $\cdots$, case E60;---, case E80; ---, case E95.

exponential-like tail, in agreement with (6.5). This result is in agreement with the findings of Vollmer et al. (2002). They performed experiments on open-channel flow over a gravel bed with the objective of investigating the effect of turbulent pressure fluctuations on the exchange of solutes between the flow in the channel and the flow inside the bed. Pressure gauges were used to measure pressure fluctuations at several heights inside the bed. The r.m.s. pressure was found to decrease exponentially inside the bed, where high-frequency oscillations were vanishing more rapidly than low-frequency oscillations.

\subsection{Vorticity fluctuations}

Figure 15 presents the r.m.s. profiles of the vorticity fluctuations for different values of the wall porosity $\epsilon_{c}$. The scale used to normalize the r.m.s. vorticities is based on the following consideration. The sum of the variances of the vorticity fluctuations can 

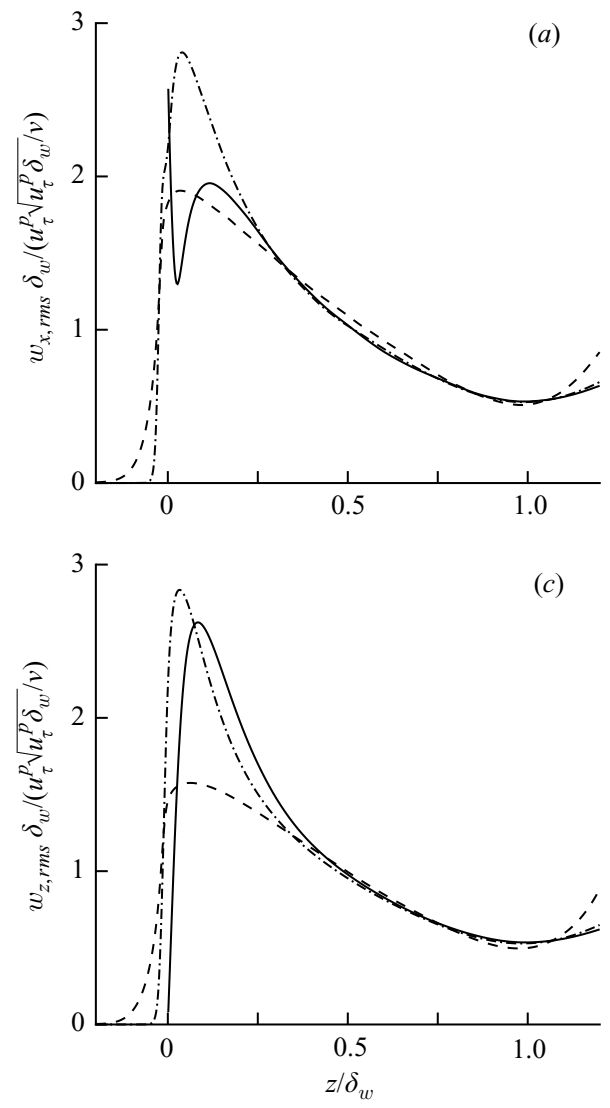

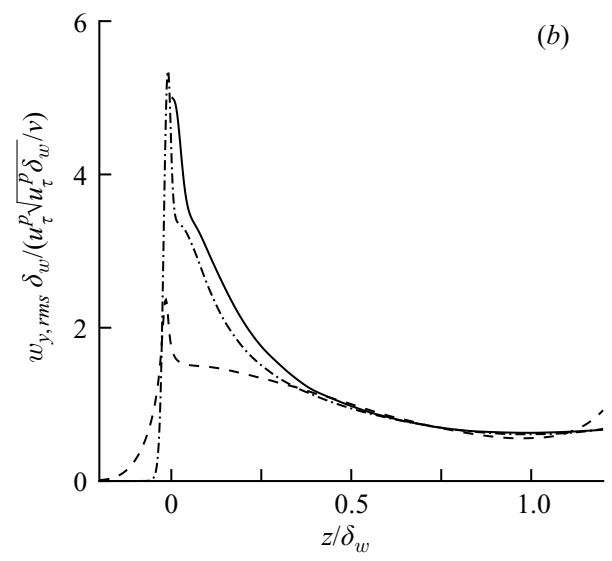

FIGURE 15. Root mean square profiles of the vorticity components normalized by $\left(u_{\tau}^{p} / \delta_{w}\right)$ $\sqrt{u_{\tau}^{p} \delta_{w} / v}$ as function of $z / \delta_{w}$. (a) Streamwise component; (b) spanwise component; $(c)$ wallnormal component. - , case E0;--.-, case E80; -.-, case E95.

be written in the following form:

$$
\overline{\left(w_{k}^{\prime}\right)^{2}}=\frac{\epsilon_{d}}{v}-\overline{\frac{\partial u_{j}^{\prime}}{\partial x_{i}} \frac{\partial u_{i}^{\prime}}{\partial x_{j}}},
$$

where the Einstein summation convention applies to all indices and where $\epsilon_{d}=$ $v \overline{\left(\partial u_{i}^{\prime} / \partial x_{j}\right)^{2}}$ is the dissipation rate of turbulent kinetic energy. In the outer region of the boundary layer above the permeable wall, the second term on the right-hand side of (6.6) is very small compared to the first term. In the same region, the dissipation rate of turbulent kinetic energy is approximately in local equilibrium with the deformation work by the Reynolds-shear stress: $\epsilon_{d} \approx \overline{u^{\prime} w^{\prime}}(\partial \bar{u} / \partial z)$. Because $\overline{u^{\prime} w^{\prime}}$ scales with $\left(u_{\tau}^{p}\right)^{2}$ and $\partial \bar{u} / \partial z$ scales with $u_{\tau}^{p} / \delta_{w}$ according to figure $8(b)$, this then implies that the r.m.s. vorticities scale with $\left(u_{\tau}^{p} / \delta_{w}\right) \sqrt{u_{\tau}^{p} \delta_{w} / \nu}$. This is substantiated by figure 15 , which shows that similarity exists for $z / \delta_{w} \gtrsim 0.35$.

In figure $15(a)$, it can be seen that the profile of $w_{x, r m s}$ has a different shape in cases E80 and E95 to that in case E0. Kim et al. (1987) associated the local minimum and maximum in $w_{x, r m s}$ in case E0 with the average position of the edge and the centre of quasi-streamwise vortices. These quasi-streamwise vortices originate from 

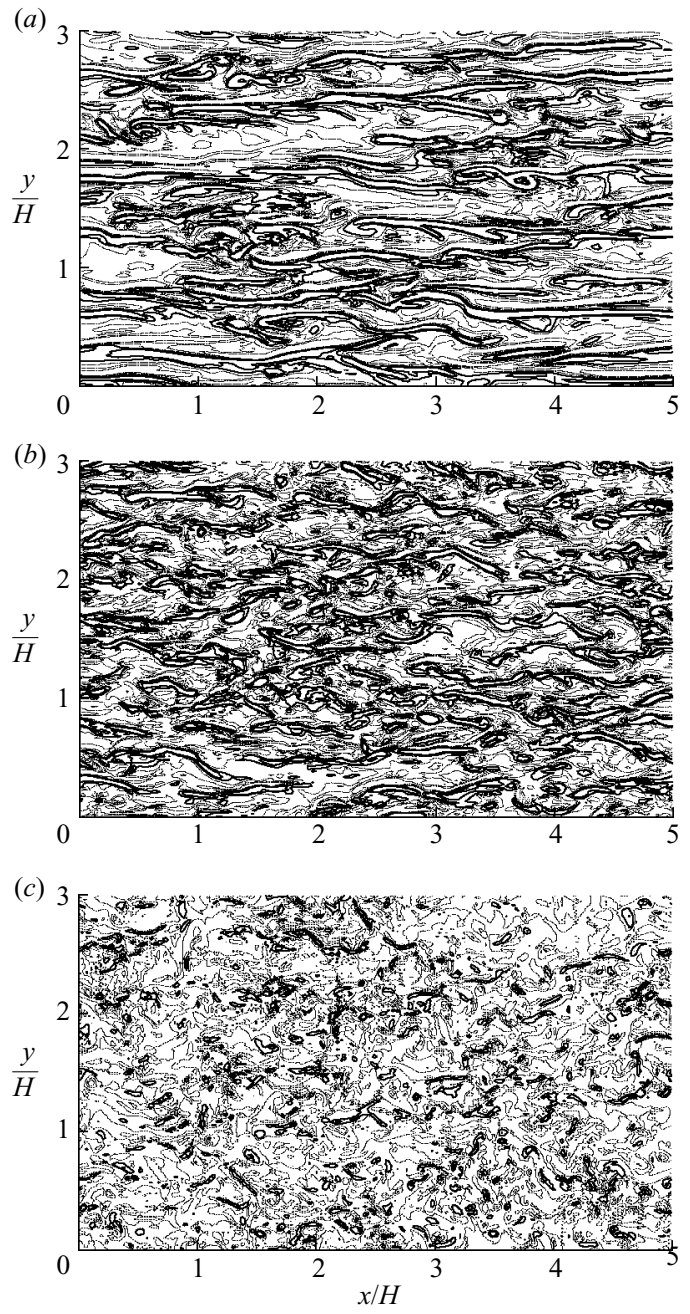

FIGURE 16. Snapshots of the wall-normal vorticity normalized by $\left(u_{\tau}^{p}\right)^{2} / v$ at $z / H=0.05$. The dotted and the bold lines are isocontours of, respectively, negative and positive vorticity at levels: $-0.2,-0.1,0,0.1,0.2$. (a) Case E0; $(b)$ case E80; $(c)$ case E95.

the presence of low- and high-speed streaks above the wall. In cases E80 and E95, no local minimum is present near the permeable wall, which indicates a change in the turbulence structure. The latter becomes obvious from figure 16, which shows snapshots of the wall-normal vorticity at $z / H=0.05$. In case E0, elongated streaky structures are present above the wall. These are the result of high- and low-speed streaks and quasi-streamwise vortices, which cause locally large values of $\left|\partial u^{\prime} / \partial y\right|$ and $\left|\partial v^{\prime} / \partial x\right|$. The snapshot of case E80 shows a more irregular structure. Figure 16(c) shows that in case E95 the streaky structures have completely vanished. For the existence of high- and low-speed streaks, a strong mean velocity gradient is required (Lee, Kim \& Moin 1990) and this condition is not satisfied above the highly permeable wall of case E95. Moreover, because of the weakening of the wall-blocking effect, in case E95, strong wall-normal velocities are present near the permeable wall and this also prevents the development of elongated streaky structures. 

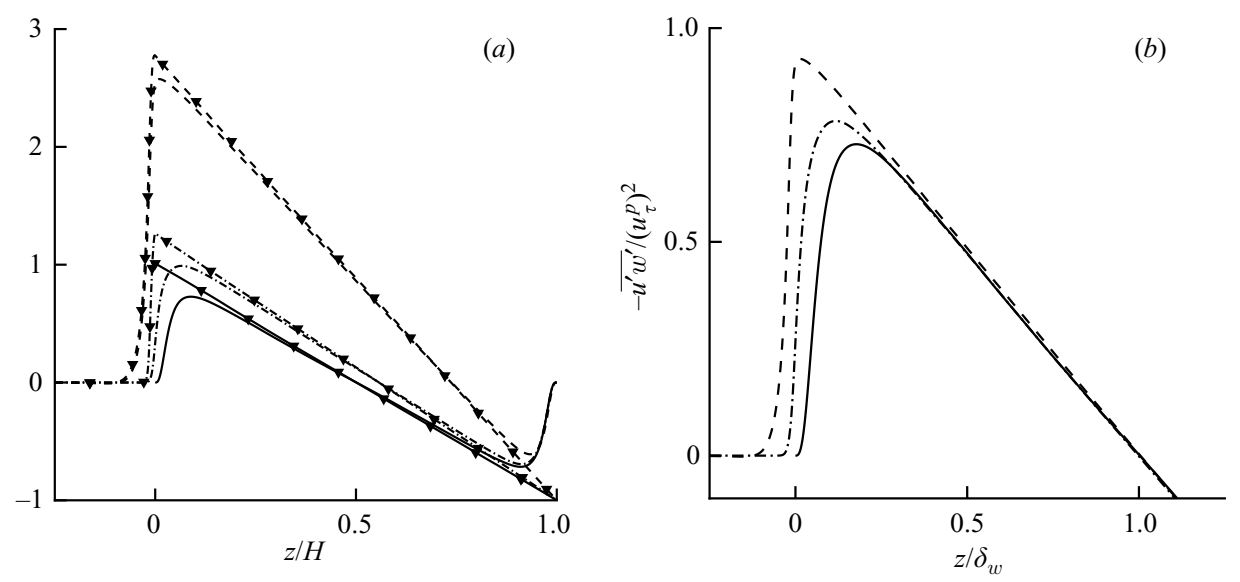

FIGURE 17. (a) Shear stress profiles normalized by $\left(u_{\tau}^{t}\right)^{2}$ as function of $z / H$. The lines with symbols are the profiles of the total shear stress $\tau_{x z} \equiv-\overline{u^{\prime} w^{\prime}}+v \mathrm{~d} \bar{u} / \mathrm{d} z$, and the lines without symbols are the profiles of the Reynolds-shear stress $-\overline{u^{\prime} w^{\prime}}$. (b) Reynolds-shear stress profiles normalized by $\left(u_{\tau}^{p}\right)^{2}$ as function of $z / \delta_{w} .-$, case E0;---, case E80; ---, case E95.

In figure 15 , it can be seen that the r.m.s. profiles of the vorticity decay faster inside the porous medium than the r.m.s. profiles of the velocity in figure 11. This is consistent with the earlier conclusion based on (6.3) that small-scale velocity fluctuations decay faster than large-scale velocity fluctuations. These large-scale velocity variations contribute to the r.m.s. of the velocity, but they do not contribute much to the r.m.s. of the vorticity.

\subsection{Reynolds and total shear stress}

Figure 17 shows profiles of the Reynolds and the total shear stress for different values of the wall porosity $\epsilon_{c}$. The total shear stress is the sum of the viscous and the Reynolds-shear stress. Figure 17( $a$ ) shows that the total shear stress is a linear function in $z$, in agreement with (4.3). The stress profiles are normalized by $\left(u_{\tau}^{t}\right)^{2}$. At the top wall the total stress is therefore equal to -1 in all cases. The total shear stress peaks at the interface with the permeable wall. In case E80 and especially in case E95, the peak is much larger than in case E0. It is observed that in case E95, the contribution from the viscous shear stress is almost negligible, which can be explained by the relaxation of the no-slip condition at the permeable wall. The strong increase in the total shear stress is caused solely by a strong increase in the Reynolds-shear stress, which in turn is caused by the weakening of the wall-blocking effect. Turbulent flow may penetrate the permeable wall $\left(w^{\prime}<0\right)$, thereby transporting fluid with relatively high streamwise momentum $\left(u^{\prime}>0\right)$ into the permeable wall. By virtue of mass conservation, fluid with relatively low streamwise momentum $\left(u^{\prime}<0\right)$ is transported from the permeable wall into the channel $\left(w^{\prime}>0\right)$.

Figure $17(b)$ presents again the profiles of the Reynolds-shear stress, but now normalized by $\left(u_{\tau}^{p}\right)^{2}$ and plotted as a function of $z / \delta_{w}$. As expected, similarity is observed in the outer region for $z / \delta_{w} \gtrsim 0.25$.

Figure 18 shows the correlation coefficient $r_{u w}$ of the velocity fluctuations $u^{\prime}$ and $w^{\prime}$, which is a measure for the efficiency of wall-normal motions in transporting streamwise momentum. Figure 18 shows that in all three cases, in a large part of the outer region, the correlation coefficient scatters around a value of 0.45 . The peak close to the permeable wall is larger for higher values of $\epsilon_{c}$, with a value of approximately 


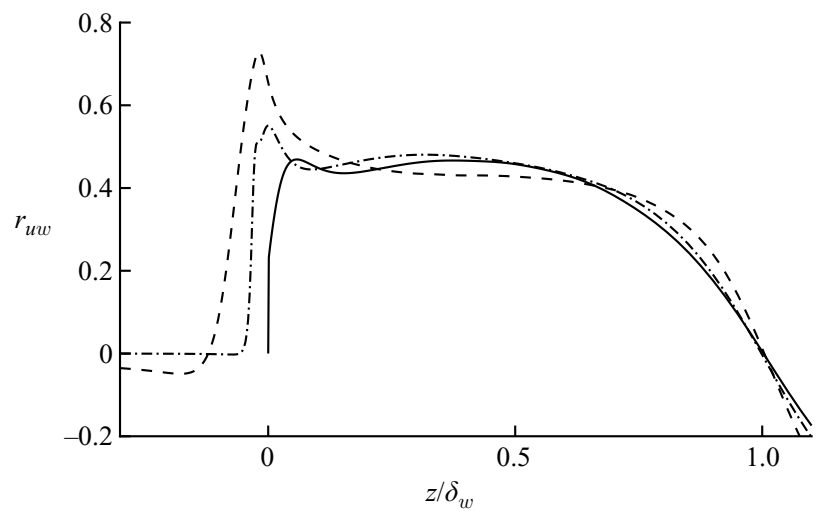

FIGURE 18. Correlation coefficient $r_{u w}=-\overline{u^{\prime} w^{\prime}} /\left(u_{r m s} w_{r m s}\right)$ as function of $z / \delta_{w}$. 一, case E0; $-\cdot-$, case E80; - - , case E95.

0.7 in case E95 against 0.5 in case E0. At the location of this peak, relatively large vortical structures are present in case E95 (see figure 4) which clearly contribute to the Reynolds-shear stress. The increase in the peak of $r_{u w}$ with increasing $\epsilon_{c}$ indicates that the increase in the Reynolds-shear stress is not just the result of a strong increase in the wall-normal turbulence intensity, but originates also from an increased efficiency of wall-normal velocity fluctuations to transport streamwise momentum. Notice the rapid decay of $r_{u w}$ inside the permeable wall. This observation is consistent with (6.3), which expresses that inside the permeable wall the velocity fluctuations are induced by pressure fluctuations. They are inactive in the sense that they do not contribute to the Reynolds-shear stress.

\subsection{Turbulent-kinetic-energy budgets}

To gain more insight into the dynamics of turbulence near a highly permeable wall,

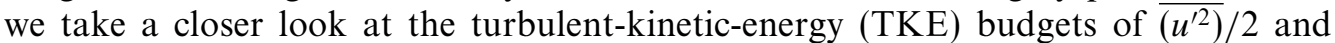
$\overline{\left(w^{\prime 2}\right)} / 2$. The transport equation for $\overline{\left(u_{\alpha}^{\prime}\right)^{2}} / 2$ can be obtained from $(5.1 a)$, which yields:

$$
\begin{aligned}
& 0=\underbrace{-\overline{u_{\alpha}^{\prime} u_{j}^{\prime}} \frac{\partial \bar{u}_{\alpha}}{\partial x_{j}}}_{W S} \underbrace{-\overline{u_{\alpha}^{\prime} \frac{\partial p^{\prime}}{\partial x_{\alpha}}}}_{V P G} \underbrace{-\frac{1}{\epsilon} \frac{\partial\left[\epsilon \frac{1}{2} \overline{\left(u_{\alpha}^{\prime}\right)^{2} u_{j}^{\prime}}\right]}{\partial x_{j}}}_{T T}+\underbrace{\frac{1}{R e_{b}} \frac{\partial^{2} \frac{1}{2} \overline{\left(u_{\alpha}^{\prime}\right)^{2}}}{\partial x_{j}^{2}}}_{V D} \underbrace{-\frac{1}{R e_{b}} \overline{\left(\frac{\partial u_{\alpha}^{\prime}}{\partial x_{j}}\right)^{2}}}_{D} \\
& +\underbrace{\frac{1}{R e_{b} \epsilon} \frac{\partial \epsilon}{\partial x_{j}} \frac{\partial \frac{1}{2} \overline{\left(u_{\alpha}^{\prime}\right)^{2}}}{\partial x_{j}}+\frac{1}{R e_{b} \epsilon} \frac{\partial^{2} \epsilon}{\partial x_{j}^{2}} \overline{\left(u_{\alpha}^{\prime}\right)^{2}}}_{\text {POR }} \underbrace{-\frac{\epsilon}{R e_{b} D a} \overline{\left(u_{\alpha}^{\prime}\right)^{2}}}_{\text {DAR }} \underbrace{-\frac{\epsilon F O}{R e_{b} D a} \overline{|\boldsymbol{u}| u_{\alpha} u_{\alpha}^{\prime}}}_{F O R},
\end{aligned}
$$

where $\alpha$ is fixed and where the Einstein summation convention applies to the subscript $j$. Term WS represents production by means of deformation work performed by the Reynolds-shear stress, term VPG is the velocity-pressure-gradient term and is responsible for a redistribution of TKE over the coordinate directions, terms $T T$ and $V D$ represent turbulent transport and viscous diffusion, respectively, and term $D$ accounts for viscous dissipation of TKE. The last three terms on the right-hand side are non-zero only inside the permeable wall. The $P O R$-terms represent transport and dissipation of TKE by local changes in the porosity. The DAR-and the FOR-term represent the transfer of resolved-scale TKE, i.e. the TKE of the volume-averaged flow, to subfilter-scale TKE by Darcy drag and Forchheimer drag, respectively. 

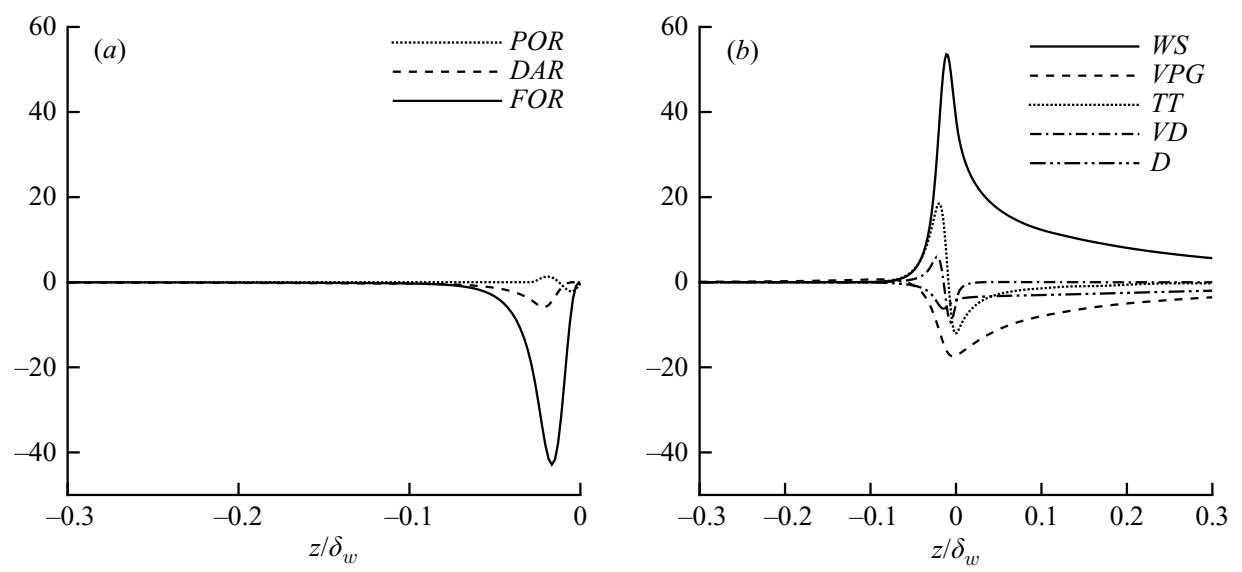

FIGURE 19. Terms in the TKE budget of $\overline{u^{\prime 2}} / 2$ for the case E95, normalized by $\left(u_{\tau}^{p}\right)^{3} / \delta_{w}$ as a function of $z / \delta_{w}$. For clarity, the terms POR, DAR and FOR, which are non-zero only inside the permeable wall, are shown in $(a)$ and the other terms in $(b)$.

Figure 19 depicts the terms in the TKE budget of $\overline{\left(u^{\prime}\right)^{2}} / 2$ for case E95. For clarity, terms POR, DAR and FOR are shown in figure $19(a)$, whereas the other terms are shown in figure $19(b)$. The terms are normalized by $\left(u_{\tau}^{p}\right)^{3} / \delta_{w}$, based on the characteristic scales for the outer region. The sum of all computed terms is negligible compared to the dominant terms.

For $z / \delta_{w} \gtrsim 0.2$, term $W S$ is in local balance with term $V P G$ and to a minor extent term $D$. Close to the permeable wall, the terms are very different from the terms in case E0. The latter are not shown here, but they can be found in Mansour, Kim \& Moin (1988). Close to the wall in case E0, term $V D$ is in balance with term $D$. Close to the highly permeable wall in case E95, however, viscous effects are of minor importance because of the relaxation of the no-slip condition. Instead, term WS is the most important source term, which originates from the weakening of the wallblocking effect, and terms $V P G$ and $T T$ are the dominant loss terms. In the top region of the permeable wall, term $W S$ reaches its top value. The most important loss term in this region is term FOR. For $z \lesssim-0.1$, term $V P G$ is in local balance with terms $D A R$ and FOR, which is in agreement with (6.3).

In figure 20, the TKE budget of $\overline{\left(w^{\prime}\right)^{2}} / 2$ is shown for case E95. A major difference with respect to case E0 is the importance of term $T T$ above the permeable wall. In a small layer around the interface it is the most dominant source term, whereas in case E0 it has about the same magnitude as term $V P G$. This is clearly related to the weakening of the wall-blocking effect near the highly permeable wall in case E95, which allows for an enhanced turbulent transport of TKE from the channel towards the permeable wall. Inside the permeable wall, term $V P G$ is balanced by terms $D A R$ and FOR, in agreement with (6.3).

\subsection{Turbulence structure}

In this section, we take a closer look at autocorrelation functions in order to investigate the turbulence structure in more detail. Figure 21 presents the spanwise autocorrelation of the streamwise velocity. Figure 21(a) corresponds to case E0, figure 21(b) to case E95. In case E0, the spanwise autocorrelation exhibits a local minimum at $y^{p+} \approx 50$ in the region close to the wall. This local minimum is usually associated with the average spanwise distance between a low-speed and a neighbouring high-speed 

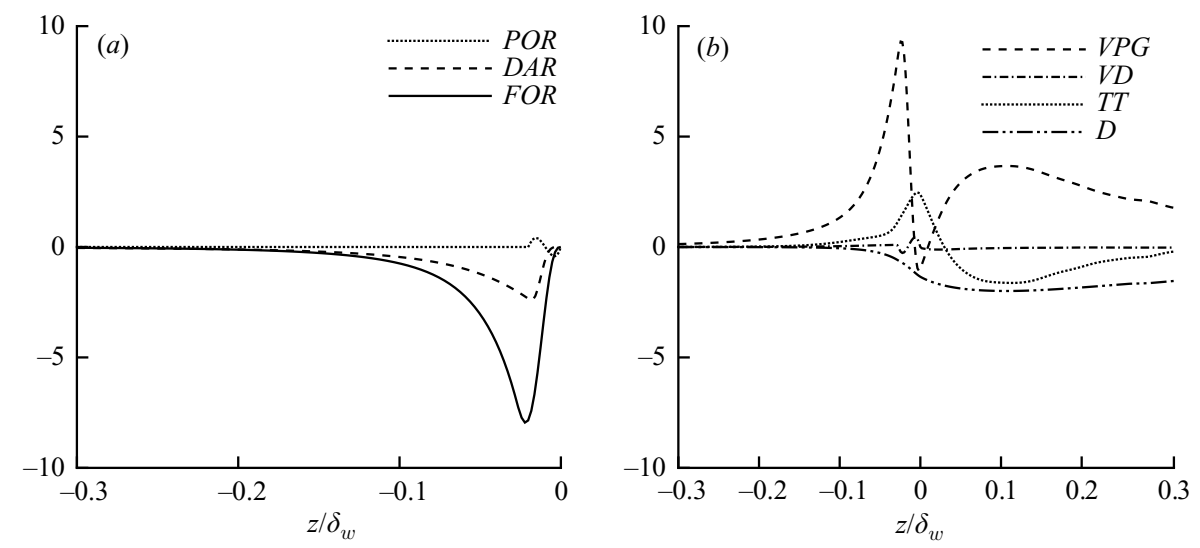

FIGURE 20. As figure 19, but for terms in the TKE budget of $\overline{w^{\prime 2}} / 2$.
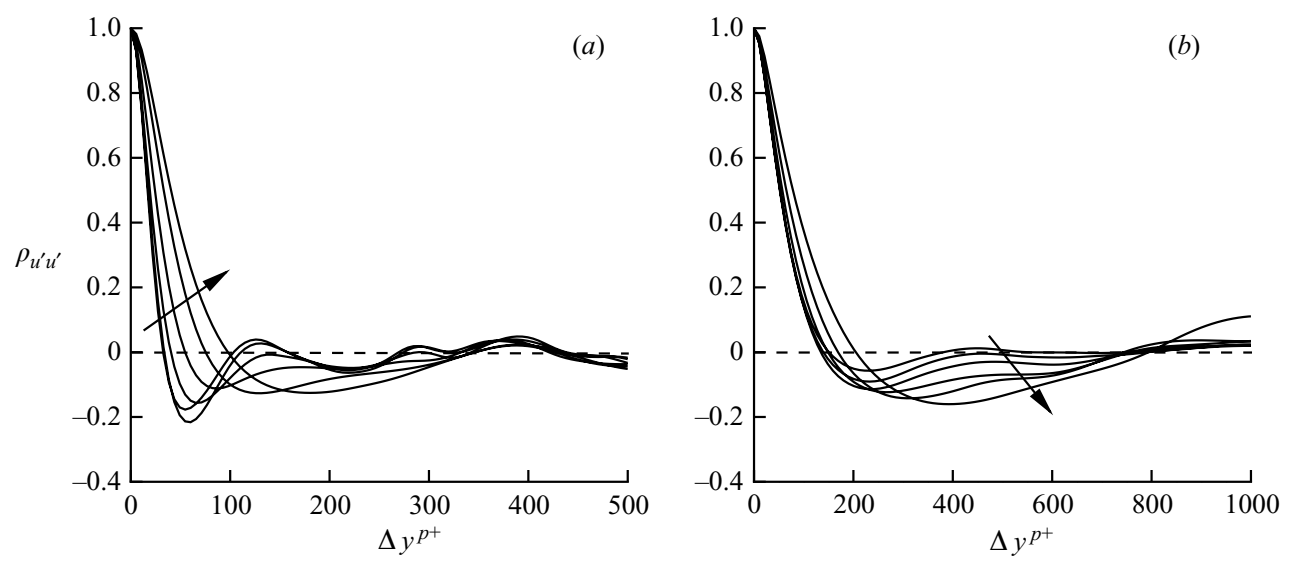

FIGURE 21. Autocorrelation $\rho_{u^{\prime} u^{\prime}}$ of the streamwise velocity as function of the spanwise spacing $\Delta y^{p+}$. The lines correspond to different wall-normal positions, increasing in the direction of the arrows: $z / H=0.01,0.035,0.075,0.14,0.24,0.49$. (a) Case E0; $(b)$ case E95.
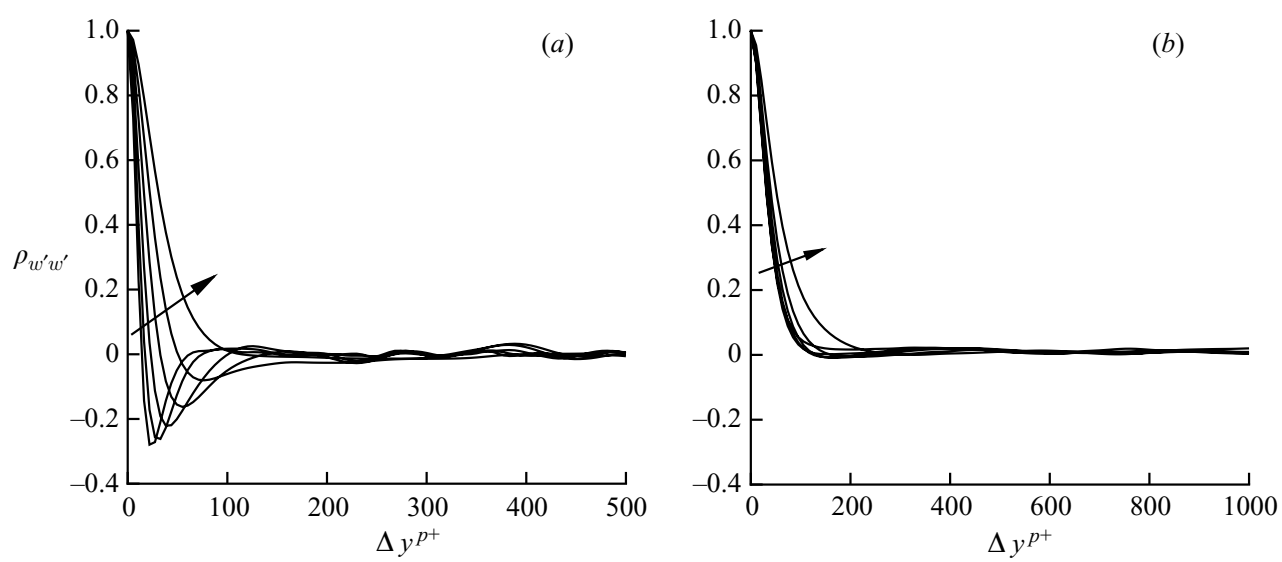

FIGURE 22. As figure 21, but for $\rho_{w^{\prime} w^{\prime}}$. 

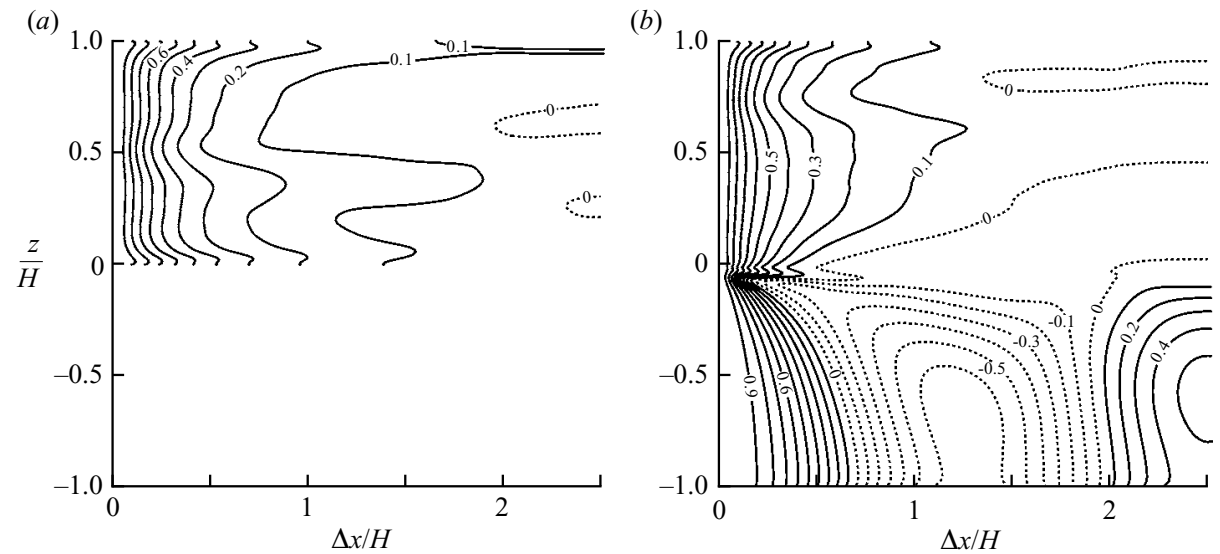

FIGURE 23. Isocontours of the autocorrelation $\rho_{u^{\prime} u^{\prime}}$ as a function of the streamwise spacing $\Delta x / H$, and shown across the permeable wall and the channel. The solid and dashed lines correspond to, respectively, positive and negative values of $\rho_{u^{\prime} u^{\prime}}$, with a step of 0.1 between two neighbouring lines. (a) Case E0; (b) Case E95.
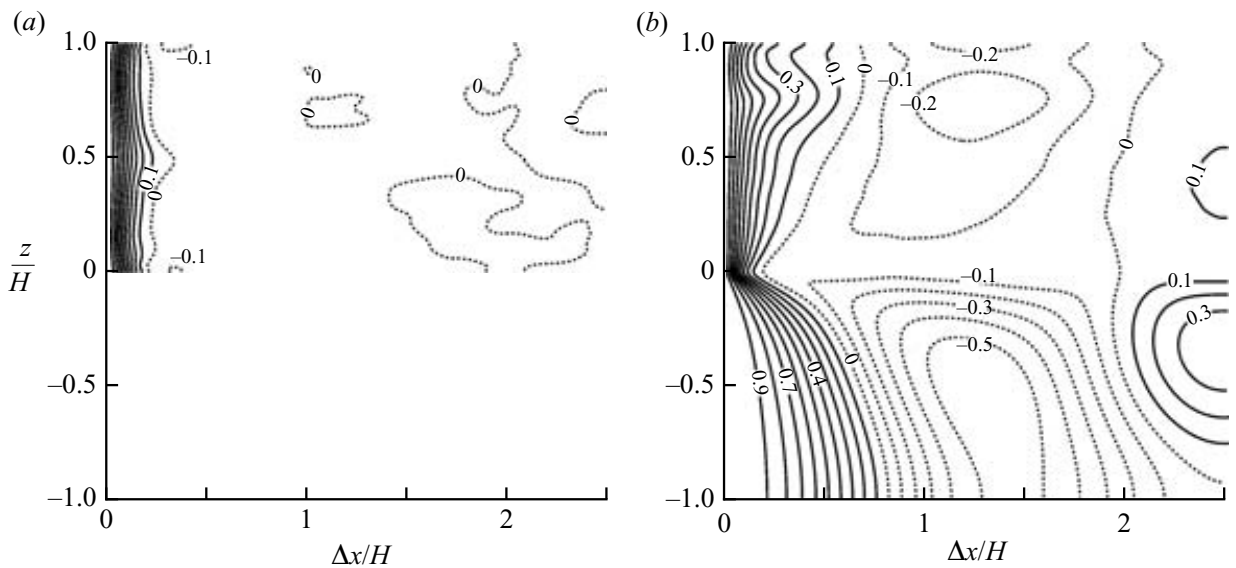

FIGURE 24. As figure 23, but for $\rho_{p^{\prime} p^{\prime}}$.

streak. The oscillations in the autocorrelation for larger spanwise spacings indicate a periodicity in the presence of the streaks. We have already observed from figure 16 that in case E95, these streaky structures have vanished. The larger correlation distance and the absence of oscillations in figure $21(b)$ confirm this. We note that the larger correlation distance is consistent with our earlier observation that turbulence near a highly permeable wall is dominated by relatively large vortical structures.

Figure 22 presents the spanwise autocorrelation of the wall-normal velocity. In case E0, a local minimum at roughly $y^{p+} \approx 30$ is observed close to the wall. This local minimum in case E0 is consistent with the presence of quasi-streamwise vortices with a typical diameter of 30 wall units. This local minimum is absent in case E95. Notice that in this case, the autocorrelation function is almost independent of the height.

In figure 23, isocontours of the streamwise autocorrelation of the streamwise velocity are shown. Notice that this is a one-dimensional correlation, but plotted across the permeable wall and the channel. Figure 23(a) corresponds to case E0 and 
figure 23(b) to case E95. In case E0, we observe a significant correlation over large distances, especially close to the walls. This behaviour is associated with the presence of streaks. The streaks have typically a length of the order of 1000 wall units. In case $\mathrm{E} 0$, this would correspond with a correlation distance of roughly $2.5 \mathrm{H}$. In case E95, the correlation distance close to the permeable wall is much smaller than in case E0, which is consistent with the absence of streaks. Inside the permeable wall, cell-like patterns are observed. According to (6.3), these originate from large-scale pressure fluctuations just above and inside the permeable wall. As can be seen from the streamwise spectrum of the pressure in figure 13(a), the most dominant streamwise wavenumber of the pressure fluctuations is $k_{x} H=4 \pi / 5$. This is also clearly reflected in figure 24(b), which shows a similar cell-like pattern for the streamwise autocorrelation of the pressure as for the streamwise velocity in figure 23(a). Notice from figure 24(b) that in case E95, there is even a significant increase in the streamwise correlation distance for the pressure close to the top wall as compared to case E0. In fact, inspection of the streamwise spectrum of the pressure revealed that $k_{x} H=4 \pi / 5$ is the dominant wavenumber throughout the flow domain.

\section{Summary and discussion}

Direct numerical simulations have been performed of turbulent flow in a plane channel with a solid top wall and a permeable bottom wall. The permeable wall is a packed bed characterized by the porosity and the mean particle diameter. In order to separate the effect of wall permeability from wall roughness, these parameters have been chosen such that in the simulations, the roughness Reynolds number is small and the permeability Reynolds number is relatively large. The VANS equations are used to describe the volume-averaged flow inside the permeable wall. The drag force is modelled by means of the Ergun equation in combination with a variable-porosity model for the interface region, whereas it is shown that subfilter-scale dispersion can be neglected. This method has been verified in a previous study (Breugem \& Boersma 2005).

Numerical results are reported of four simulations, for which only the wall porosity $\epsilon_{c}$ in the homogeneous porous region is varied. A key parameter is the permeability Reynolds number $R e_{K}$. This is the ratio of the length scale of the drag, $\propto \sqrt{K}$, to the viscous length scale $v / u_{\tau}$. This number can also be interpreted as the ratio of the effective pore diameter to the characteristic length scale of the viscous sublayers over the individual wall elements. Similar to the classification of rough walls, a highly permeable wall is classified as a permeable wall near which viscous effects are of minor importance, which corresponds to $R e_{K} \gg 1$. Just above such a wall, turbulent transport of MKE is counterbalanced by the work performed by the Reynolds-shear stress, and just below the wall interface, the Forchheimer drag force is an additional source of dissipation. For $R e_{K} \ll 1$, a permeable wall is effectively impermeable. Close to such a wall, viscous diffusion of MKE is counterbalanced by viscous dissipation.

In all simulations, the logarithmic law could be fitted to the mean velocity in the boundary layer over the permeable wall. The values found from this fit for the slope $(1 / \lambda)$, the displacement height $(d)$ and the equivalent roughness height $\left(z_{0}\right)$, were very different from the literature values for fully rough walls and suggest a relation to the permeability of the wall. Furthermore, the large values for $d$ could not be explained by the modified Jackson model. It cannot be excluded that the fitted values are obscured by a low-Reynolds-number effect. In our simulations, the highest Reynolds 
number based on the friction velocity and the boundary-layer thickness is found for case E95: $R e_{\delta}^{p}=498$ (see table 2). This number may still be considered as fairly small, and one may doubt whether the conditions are satisfied for the existence of a logarithmic overlap region between the inner and outer scaling regions. On the other hand, in case E0, the Reynolds number $R e_{\delta}^{p}$ is almost three times smaller, but $\lambda=0.4$ was found to be in agreement with the generally accepted value of 0.4 for the von Kármán constant. Besides, as can be seen from figure 6(a), the extent of the logarithmic layer in case E0 is also significantly smaller than in case E95 (both in wall units and as a fraction of the boundary-layer thickness). At this moment we have no explanation for the unusual fit values, and we recommend experiments at high(er) Reynolds numbers to investigate this further.

It is found that the mean velocity profile decreases exponentially inside a permeable wall. For a highly permeable wall, the exponential decay is governed by the balance between turbulence diffusion of momentum into the permeable wall on the one hand and the removal of momentum by Forchheimer drag on the other.

It is shown that the structure and dynamics of turbulence near a highly permeable wall are different compared to an effectively impermeable wall. Above a highly permeable wall, no low- and high-speed streaks and the associated quasi-streamwise vortices are present, which are typical features of turbulence near a solid wall. The absence of streaks can be explained by a strong reduction in mean shear, which in turn originates from the relaxation of the no-slip condition. Furthermore, turbulent transport across the wall interface, which is due to a weakening of the wall-blocking effect, also prevents the development of elongated streaks. Turbulence near a highly permeable wall is dominated by relatively large vortical structures, which are responsible for the exchange of momentum between the top layer of the porous medium and the channel. These vortical structures originate from a Kelvin-Helmholtz type of instability of the mean velocity profile, which exhibits a point of inflection, as hypothesized by Raupach et al. (1991) and Finnigan (2000). The exchange of momentum between the channel region and the top layer of the permeable wall induces a strong increase in the Reynolds-shear stress relative to the case of a solid wall. Near a highly permeable wall, a significant increase is observed in the correlation coefficient $r_{u w}$. This indicates that the vortical structures near the permeable wall are very efficient in transporting streamwise momentum in the wall-normal direction.

The strong increase in the Reynolds-shear stress explains the strong increase in the skin friction at a highly permeable wall as compared to an impermeable wall. This result is in contrast to the decrease in skin friction found in the simulations of Hahn et al. (2002). They, however, assumed that the flow near the permeable wall is essentially laminar, and, because wall permeability causes a decrease in the viscous wall shear stress, they therefore found a decrease in the skin friction. As discussed in $\S 1$, there is experimental evidence that wall permeability causes an increase in skin friction in the turbulent-flow regime (Zagni \& Smith 1976; Zippe \& Graf 1983; Kong \& Schetz 1982), whereas it causes a decrease in the skin friction in the laminar-flow regime (Beavers \& Joseph 1967).

The absence of streaks above a highly permeable wall is consistent with a decrease in the peak of the streamwise r.m.s. velocity. Despite the increase in the peak of the spanwise and wall-normal r.m.s. velocities, owing to a relaxation of the no-slip and no-penetration conditions, the peak in the turbulent kinetic energy is therefore smaller. Inside the permeable wall, the r.m.s. profiles exhibit an exponential-like tail. It is shown that the turbulence motions inside the wall are inactive in the sense that they are induced by pressure fluctuations. They do not contribute to the Reynolds-shear 
stress. Furthermore, inside the permeable wall, small-scale fluctuations decrease more rapidly than large-scale fluctuations.

A large increase is found in the r.m.s. of the pressure above a highly permeable wall, which is related to transport of fluid across the wall interface and hence to a weakening of the wall-blocking effect. Besides an increase in the r.m.s. of the pressure, also the velocity-pressure-gradient term in the TKE budget for $\overline{u^{\prime 2}} / 2$ gained in importance near the permeable wall. In the channel region, this term is responsible for the transfer of streamwise TKE to spanwise and wall-normal TKE. Near a highly permeable wall the velocity-pressure-gradient term is the most dominant loss term of streamwise TKE, whereas near a solid wall, viscous dissipation is the most important loss term.

Some statistics exhibit similarity in the outer region, such as the streamwise r.m.s. velocity, the r.m.s. vorticities and the Reynolds-shear stress. This substantiates the wall similarity hypothesis. However, other statistics show significant deviations from similarity, such as the spanwise and wall-normal r.m.s. velocities and the r.m.s. of the pressure. This could be a low-Reynolds-number effect, but Kroogstad et al. (1992) have suggested that the departure from similarity in the outer region originates from the weakening of the wall-blocking effect. By means of strong ejection and sweep events, fluid can be transported over a large vertical distance. As a consequence, the outer region is able to communicate with the inner region and this might explain the observed departures from similarity.

This work was sponsored by the National Computing Facilities Foundation (NCF) for the use of supercomputer facilities, with financial support from the Netherlands Organization for Scientific Research (NWO). The authors would like to thank Professor F.T.M. Nieuwstadt and Professor J.C.R. Hunt for discussions and comments on the manuscript.

\section{Appendix A. Closure problem for the subfilter-scale stress}

The nature of the subfilter-scale stress in a porous medium is more complex than the subgrid-scale stress in LES. In a homogeneous fluid region, subgrid-scale turbulent motions contribute to diffusion of resolved-scale momentum when a resolved-scale strain field is present. We refer to this as turbulent dispersion. In a porous medium, the fluid is forced to move around the solid obstacles, and this causes an additional contribution to diffusion of volume-averaged momentum. This is known as mechanical dispersion (Bear 1988). Both types of dispersion are shown in figure 25. In this section, we argue that in general both the mechanical and the turbulent subfilter-scale stress have a negligible influence on the volume-averaged flow field.

From figure 25, it can be inferred that mechanical and turbulent dispersion take place only when a volume-averaged strain field is present. This motivates a customary parameterization of the subfilter-scale stress according to:

$$
\tau_{i j}=-\left(v_{p}+v_{t}\right)\left(\frac{\partial\left\langle u_{i}\right\rangle}{\partial x_{j}}+\frac{\partial\left\langle u_{j}\right\rangle}{\partial x_{i}}\right),
$$

where $v_{p}$ and $v_{t}$ are, respectively, the mechanical and the turbulent viscosity. The summation of the two viscosities in (A 1) is allowed, provided that they do not mutually correlate.

When a fluid element with a velocity $\tilde{\boldsymbol{u}}$ approaches a solid obstacle of diameter $d_{p}$, it has to move around it. From this consideration we estimate the mechanical 


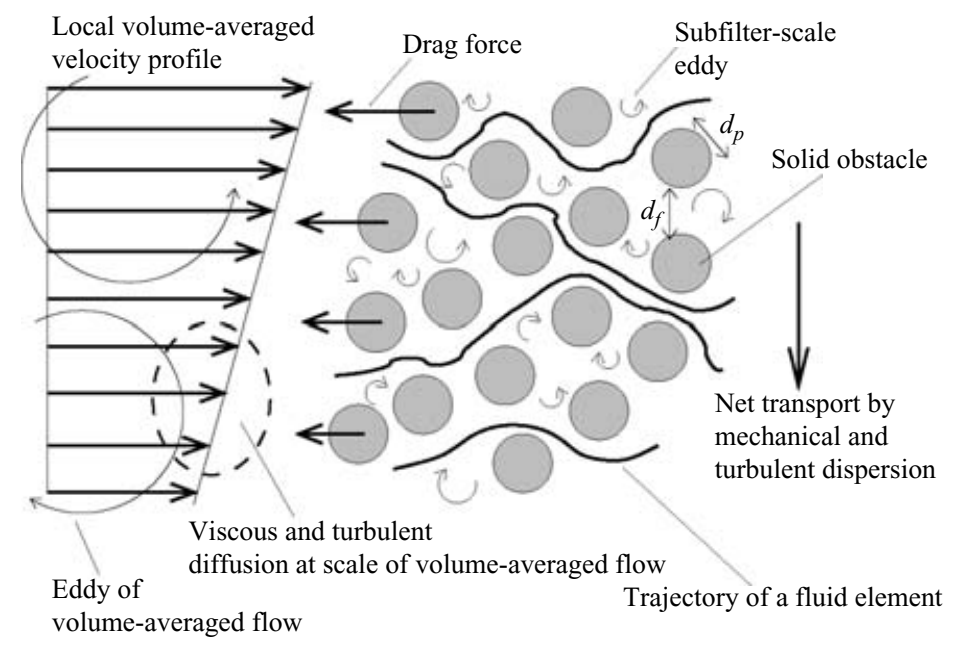

FIGURE 25. Illustration of turbulent and mechanical dispersion in a porous medium. The figure also shows the presence of large-scale eddies, which are responsible for turbulent diffusion at the scale of the volume-averaged flow, and the drag force that the solid obstacles exert on the volume-averaged flow.

viscosity by:

$$
v_{p}=c_{p} d_{p} \sqrt{e},
$$

where $e \equiv\left\langle u_{i} u_{i}\right\rangle / 2-\left\langle u_{i}\right\rangle\left\langle u_{i}\right\rangle / 2 \approx\left\langle\tilde{u}_{i} \tilde{u}_{i}\right\rangle / 2$ is the subfilter-scale kinetic energy, and $c_{p}$ is a coefficient that depends on the structure of the porous medium. It is expected that for porous media for which solid obstacles are aligned relative to each other, $c_{p}$ will be very small, whereas this coefficient will be larger for porous media where the solid obstacles are staggered or randomly distributed. The turbulent viscosity is estimated by a similar consideration. It is assumed that the typical length scale of the subfilter-scale eddies is proportional to the typical pore diameter $d_{f}$. This yields:

$$
v_{t}=c_{t} d_{f} \sqrt{e},
$$

where $c_{t}$ is a coefficient that depends on the characteristic Reynolds number for the flow in the pores. For low Reynolds numbers, the flow in the pores is laminar and consequently the coefficient $c_{t}$ is very small, but for large Reynolds numbers, the flow in the pores is turbulent and $c_{t}$ is of order unity. This was recognized by Uittenbogaard (2003), who developed a $k-\epsilon$ turbulence model for flow over aquatic vegetation, where a parameterization similar to (A 3) was employed for the turbulent subfilter-scale stress inside the vegetation layer.

Let us consider two extreme cases, namely the case where turbulent dispersion dominates over mechanical dispersion, and the opposite case where mechanical dispersion is dominant.

\section{(i) Mechanical dispersion dominates strongly over turbulent dispersion}

Mechanical dispersion dominates strongly over turbulent dispersion for $d_{f} / d_{p} \ll 1$ and/or a low characteristic Reynolds number for the flow in the pores, $|\langle\boldsymbol{u}\rangle| d_{f} / v \ll 1$. These conditions are often encountered in packed beds. In this case, mechanical dispersion turns out to be of minor importance as compared to the influence of drag on the volume-averaged flow. From (2.3), it can be inferred that for $d_{f} / d_{p} \ll 1$, the 
subfilter-scale kinetic energy will be of the order of the kinetic energy of the volumeaveraged flow: $e=O\left(\left\langle u_{i}\right\rangle^{2} / 2\right)$. The dispersion term in the VANS equation (2.7a) can therefore be estimated by:

$$
\frac{1}{\epsilon} \frac{\partial \epsilon \tau_{i j}}{\partial x_{j}}=O\left(\frac{c_{p} d_{p}|\langle\boldsymbol{u}\rangle|\left\langle u_{i}\right\rangle}{L_{u}^{2}}\right),
$$

where $L_{u}$ is a typical length scale of the volume-averaged flow. An estimate for the drag term is found from (2.8). Neglecting Forchheimer drag and modelling the permeability tensor according to $(2.9 a)$, the drag term in $(2.7 a)$ reads:

$$
f_{i}=-v \frac{180(1-\epsilon)^{2}}{d_{p}^{2} \epsilon^{2}}\left\langle u_{i}\right\rangle \text {. }
$$

The ratio of the dispersion term to the drag term in $(2.7 a)$ can thus be estimated by:

$$
\left|\frac{1}{f_{i}} \frac{1}{\epsilon} \frac{\partial \epsilon \tau_{i j}}{\partial x_{j}}\right|=O\left(\frac{c_{p}}{180} \frac{\epsilon\left[1-\epsilon^{1 / 3}\right]^{3}}{[1-\epsilon]^{2}}\left[\frac{|\langle\boldsymbol{u}\rangle| d_{f}}{v}\right]\left[\frac{d_{f}}{L_{u}}\right]^{2}\right) \ll 1,
$$

in which we used the estimate that $\left(d_{p} / d_{f}\right)^{3}=O\left(\left[1-\epsilon^{1 / 3}\right]^{3} / \epsilon\right)$. This result shows that if mechanical dispersion dominates over turbulent dispersion, then the influence of drag on the volume-averaged flow is much more important than mechanical dispersion.

(ii) Turbulent dispersion dominates strongly over mechanical dispersion

Turbulent dispersion dominates strongly over mechanical dispersion when both the Reynolds number $|\langle\boldsymbol{u}\rangle| d_{f} / v$ and the ratio $d_{f} / d_{p}$ are large. The last condition is equivalent to a very high porosity, close to unity, which can be encountered in a forest. In this case, the turbulent subfilter-scale stress can be neglected if the turbulent viscosity $v_{t}$ is much smaller than the eddy viscosity $v_{T}$ of the turbulent volumeaveraged flow. The difference between the eddies of the subfilter-scale flow and of the volume-averaged flow is illustrated in figure 25. The value of $v_{T}$ can be estimated by $v_{T}=\mathscr{L} \mathscr{U}$, where $\mathscr{L}$ is the mixing length and $\mathscr{U}=\left[\overline{\left\langle u_{i}\right\rangle^{\prime}\left\langle u_{i}\right\rangle^{\prime}} / 2\right]^{1 / 2}$ is a characteristic velocity scale of the large-scale eddies. The ratio of $v_{t}$ to $v_{T}$ is given by:

$$
\frac{v_{t}}{v_{T}}=c_{t} \frac{d_{f}}{\mathscr{L}} \frac{\sqrt{e}}{\mathscr{U}} .
$$

If $d_{f} / \mathscr{L} \ll 1$ holds, then also $\sqrt{e} / \mathscr{U} \ll 1$ holds, because the large eddies are much more energetic than the small eddies. Thus, when $d_{f} / \mathscr{L} \ll 1$ holds, the turbulent subfilter-scale stress can be neglected. This is typically the case in a forest in which turbulence is dominated by scales of the order of the height of the forest (Finnigan 2000). Turbulent dispersion can be neglected because the height of the forest (scale for $\mathscr{L}$ ) is much larger than the typical length scale of the individual forest elements (scale for $d_{f}$ ).

The above analysis suggests that for both extreme cases the effect of dispersion on the volume-averaged flow field is generally negligible. This suggests that subfilter-scale dispersion may always be neglected, and also in cases where mechanical and turbulent dispersion are equally important.

\section{Appendix B. Modification of Jackson model for presence of pressure gradient}

For boundary-layer flow over a porous wall in the absence of a mean pressure gradient, Jackson (1981) proposed that the displacement height $d$ in the logarithmic 
law is the level at which the height-averaged drag force acts. This suggests that $d$ can be calculated from:

$$
d=-\frac{\int z f \mathrm{~d} z}{\int f \mathrm{~d} z},
$$

where $f$ is the height-dependent drag force and where the integral extends over the entire height of the porous medium. We remark that in the absence of a mean pressure gradient, the drag force is balancing viscous and turbulent diffusion. As shown by Jackson, $d$ could therefore also be interpreted as the displacement thickness of the total shear stress in the porous medium.

In the presence of a mean pressure gradient, the drag force is not only balancing diffusion of momentum, but also the mean pressure gradient. This balance is expressed by the Reynolds-averaged momentum equation for the streamwise direction, which can be obtained from $(5.1 a)$ :

$$
-\frac{1}{R e_{b}} \frac{\epsilon}{D a} \bar{u}-\frac{1}{R e_{b}} \frac{\epsilon F o}{D a} \overline{|\boldsymbol{u}| u}-\frac{1}{R e_{b}} \frac{1}{\epsilon} \frac{\partial \epsilon}{\partial z} \frac{\partial \bar{u}}{\partial z}-\frac{\mathrm{d} \bar{p}}{\mathrm{~d} x}=\frac{1}{\epsilon} \frac{\partial}{\partial z}\left[\epsilon \overline{u^{\prime} w^{\prime}}-\frac{1}{R e_{b}} \frac{\partial \epsilon \bar{u}}{\partial z}\right] .
$$

We now define the left-hand side of (B2) as the effective drag force $f_{e}$ :

$$
f_{e} \equiv f-\frac{\mathrm{d} \bar{p}}{\mathrm{~d} x}=-\frac{1}{R e_{b}} \frac{\epsilon}{D a} \bar{u}-\frac{1}{R e_{b}} \frac{\epsilon F o}{D a} \overline{|\boldsymbol{u}| u}-\frac{1}{R e_{b}} \frac{1}{\epsilon} \frac{\partial \epsilon}{\partial z} \frac{\partial \bar{u}}{\partial z}-\frac{\mathrm{d} \bar{p}}{\mathrm{~d} x} .
$$

Consistent with the interpretation of $d$ as the displacement thickness of the total shear stress inside the porous medium, we propose the following modification of (B 1) to account for the presence of a mean pressure gradient:

$$
d=d_{1}+d_{2}, \quad d_{2}=-\frac{\int\left(z+d_{1}\right) f_{e} \mathrm{~d} z}{\int f_{e} \mathrm{~d} z} \text { with } z<-d_{1},
$$

where $z=-d_{1}$ is the height at which $f_{e}=0$. In a way, $z=-d_{1}$ could be interpreted as the location of the effective interface between channel and permeable wall, where for $z<-d_{1}$ the effective drag force is responsible for absorption of momentum. Note that $d$ approaches the correct limits when $K_{c} \rightarrow 0$ and $K_{c} \rightarrow \infty$. When $K_{c} \rightarrow \infty$, i.e. no solid obstacles, the effective drag force becomes equal to $-\mathrm{d} \bar{p} / \mathrm{d} x$. Consequently, $d=d_{1}=h$, where $h$ is the whole thickness of the permeable wall, which is the correct value as in this case, the impermeable wall at $z=-h$ is the actual wall. When $K_{c} \rightarrow 0$, i.e. when the permeable wall is completely impermeable, there is no flow inside the permeable wall and hence the effective drag force is equal to zero. This leads to the correct limit of $d=0$.

\section{REFERENCES}

Bear, J. 1988 Dynamics of Fluids in Porous Media. Dover.

BeAvers, G. S. \& JosePH, D. D. 1967 Boundary conditions at a naturally permeable wall. J. Fluid Mech. 30, 197-207.

Bird, R. B., Stewart, W. E. \& Lightfoot, E. N. 2002 Transport Phenomena. John Wiley.

Breugem, W. P. 2004 The influence of wall permeability on laminar and turbulent flows. Theory and simulations. PhD thesis, Delft University of Technology, Delft. 
Breugem, W. P. \& Boersma, B. J. 2005 Direct numerical simulations of turbulent flow over a permeable wall using a direct and a continuum approach. Phys. Fluids 17, 025103: 1-15.

Breugem, W. P., Boersma, B. J. \& Uittenbogand, R. E. 2004 Direct numerical simulation of plane channel flow over a 3D Cartesian grid of cubes. In Proc. Intl Conf. on Applications of Porous Media (ed. A. H. Reis \& A. F. Miguel), pp. 27-34. Évora Geophysics Center, Évora, Portugal.

Brinkman, H. C. 1948 A calculation of the viscous force exerted by a flowing fluid on a dense swarm of particles. Appl. Sci. Res. A 1, 27-34.

De Lemos, M. J. S. \& Pedras, M. H. J. 2000 Simulation of turbulent flow through hybrid porous medium-clear fluid domains. In Proc. ASME Heat Transfer Division, HTD- 366-5.

Drazin, P. G. \& ReID, W. H. 1981 Hydrodynamic Stability. Cambridge University Press.

Dwyer, M. J., Patton, E. G. \& Shaw, R. H. 1997 Turbulent kinetic energy budgets from a large-eddy simulation of airflow above and within a forest canopy. Boundary-Layer Met. 84, 23-43.

Fand, R. M., Kim, B. Y. K., Lam, A. C. C. \& Phan, R. T. 1987 Resistance to the flow of fluids through simple and complex porous media whose matrices are composed of randomly packed spheres. J. Fluids Engng 109, 268-274.

Finnigan, J. 2000 Turbulence in plant canopies. Annu. Rev. Fluid Mech. 32, 519-571.

GraY, W. G. 1975 A derivation of the equations for multi-phase transport. Chem. Engng Sci. 30, 229-233.

Hahn, S., Je, J. \& Chol, H. 2002 Turbulent channel flow with permeable walls. J. Fluid Mech. 450, $259-285$.

HinZe, J. O. 1975 Turbulence. McGraw-Hill.

Hunt, J. C. R. \& Durbin, P. A. 1999 Perturbed vortical layers and shear sheltering. Fluid Dyn. Res. 24, 375-404.

JACKSON, P. S. 1981 On the displacement height in the logarithmic velocity profile. J. Fluid Mech. 111, $15-25$.

Kececioglu, I. \& Jiang, Y. 1994 Flow through porous media of packed spheres saturated with water. J. Fluids Engng 116, 164-170.

Kim, J., Morn, P. \& Moser, R. 1987 Turbulence statistics in fully developed channel flow at low Reynolds number. J. Fluid Mech. 177, 133-166.

Kong, F. Y. \& SchetZ, J. A. 1982 Turbulent boundary layer over porous surfaces with different surface geometries. AIAA Paper 82-0030.

Kro̊gstad, P.-A. \& Antonia, R. A. 1999 Surface roughness effects in turbulent boundary layers. Exps Fluids 27, 450-460.

Kro̊gstad, P.-A., Antonia, R. A. \& Browne, L. W. B. 1992 Comparison between rough- and smooth-wall turbulent boundary layers. J. Fluid Mech. 245, 599-617.

Lage, J. L., Antohe, B. V. \& Nield, D. A. 1997 Two types of nonlinear pressure-drop versus flow-rate relation observed for saturated porous media. J. Fluids Engng 119, 700706.

Larson, R. E. \& Higdon, J. J. L. 1986 Microscopic flow near the surface of two-dimensional porous media. Part 1. Axial flow. J. Fluid Mech. 166, 449-472.

LARSON, R. E. \& Higdon, J. J. L. 1987 Microscopic flow near the surface of two-dimensional porous media. Part 1. Transverse flow. J. Fluid Mech. 178, 119-136.

LeE, M. J., Kim, J. \& Moin, P. 1990 Structure of turbulence at high shear rate. J. Fluid Mech. 216, 561-583.

LeE, S. L. \& Yang, J. H. 1997 Modeling of Darcy-Forchheimer drag for fluid flow across a bank of circular cylinders. Intl J. Heat Mass Transfer 40, 3149-3155.

Lu, T. J., Stone, H. A. \& Ashby, M. F. 1998 Heat transfer in open-cell metal foams. Acta Material. 46, 3619-3635.

MA, H. \& Ruth, W. 1993 The microscopic analysis of high Forchheimer number flow in porous media. Transp. Porous Media 13, 139-160.

MacDonald, I. F., El-Sayed, M. S., Mow, K. \& Dullien, F. A. L. 1979 Flow through porous media: the Ergun equation revisited. Indust. Engng Chem. Fund. 18, 199-208.

Mansour, N. N., KIm, J. \& MoIN, P. 1988 Reynolds-stress and dissipation-rate budgets in a turbulent channel flow. J. Fluid Mech. 194, 15-44. 
NePF, H. M. 1999 Drag, turbulence, and diffusion in flow through emergent vegetation. Water Resour. Res. 35, 479-489.

OChOA-TAPia, J. A. \& Whitaker, S. 1995 Momentum transfer at the boundary between a porous medium and a homogeneous fluid - II. Comparison with experiment. Intl J. Heat Mass Transfer 38, 2647-2655.

Pedras, M. H. J. \& De Lemos, M. J. S. 2001 Macroscopic turbulence modeling for incompressible flow through deformable porous media. Intl J. Heat Mass Transfer 44, 1081-1093.

Perot, B. \& Moin, P. 1995 Shear-free turbulent boundary layers. Part 1. Physical insights into near-wall turbulence. J. Fluid Mech. 295, 199-227.

QuintaRd, M. \& Whitaker, S. 1994 Transport in ordered and disordered porous media II: Generalized volume averaging. Transp. Porous Media 14, 179-206.

Raupach, M. R., Antonia, R. A. \& Rajagopalan, S. 1991 Rough-wall turbulent boundary layers. Appl. Mech. Rev. 44, 1-25.

Ruff, J. F. \& Gelhar, L. W. 1972 Turbulent shear flow in porous boundary. J. Engng Mech. Div. 98, 975-991.

Sahraoui, M. \& Kaviany, M. 1992 Slip and no-slip velocity boundary conditions at interface porous, plain media. Intl J. Heat Mass Transfer 35, 927-943.

SÉro-Guillaume, O. \& Margerit, J. 2002 Modeling forest fires. Part I: a complete set of equations derived by extended irreversible thermodynamics. Intl J. Heat Mass Transfer 45, 1705-1722.

Shaw, R. H. \& Schumann, U. 1992 Large-eddy simulation of turbulent flow above and within a forest. Boundary-Layer Met. 61, 47-64.

Silva, R. A. \& De Lemos, M. J. S. 2003 Turbulent flow in a channel occupied by a porous layer considering the stress jump at the interface. Intl J. Heat Mass Transfer 46, 5113-5121.

Tennekes, H. \& Lumley, J. L. 1999 A First Course in Turbulence. MIT Press.

UittenbogaARD, R. E. 2003 Modeling turbulence in vegetated aquatic flows. Proc. Intl Workshop on Riparian Forest, 20-22 February, Trento, Italy.

VAfAI, K. 1984 Convective flow and heat transfer in variable-porosity media. J. Fluid Mech. 147, $233-259$.

Vollmer, S., Francisco de los Santos, R., Daebel, H. \& Kühn, G. 2002 Micro scale exchange processes between surface and subsurface water. J. Hydrol. 269, 3-10.

WATANABE, T. 2004 Large-eddy simulation of coherent turbulence structures associated with scalar ramps over plant canopies. Boundary-Layer Met. 112, 307-341.

Wesseling, P. 2001 Principles of Computational Fluid Dynamics. Springer.

Whitaker, S. 1996 Advances in theory of fluid motion in porous media. Indust. Engng Chem. 61, $14-28$.

Whitaker, S. 1996 The Forchheimer equation: a theoretical development. Transp. Porous Media 25, $27-61$.

Whitaker, S. 1999 The Method of Volume Averaging. Kluwer.

Wilson, J. D. 1988 A second-order closure model for flow through vegetation. Boundary-Layer Met. 42, 371-392.

Yaglom, A. M. 1979 Similarity laws for constant-pressure and pressure-gradient turbulent wall flows. Annu. Rev. Fluid Mech. 11, 505-540.

ZaGni, A. F. E. \& SMith, K. V. H. 1976 Channel flow over permeable beds of graded spheres. J. Hydraul. Div. 102, 207-222.

Zick, A. A. \& Homsy, G. M. 1982 Stokes flow through periodic arrays of spheres. J. Fluid Mech. $115,13-26$.

ZIPPE, H. J. \& GRAF, W. H. 1983 Turbulent boundary-layer flow over permeable and non-permeable rough surfaces. J. Hydraul. Res. 21, 51-65. 